\title{
Superfluid Neutrons in the Core of the Neutron Star in Cassiopeia A
}

\section{Dany Page*}

Instituto de Astronomía, Universidad Nacional Autónoma de México, Mexico, DF 04510, Mexico

E-mail: pageeastro.unam.mx

\section{Madappa Prakash}

Department of Physics and Astrononmy, Ohio University,

Athens, OH 45701-2979, USA

E-mail: prakash@harsha.phy.ohiou.edu

\section{James M. Lattimer}

Department of Physics and Astronomy, State University of New York at Stony Brook,

Stony Brook, NY 11794-3800, USA

E-mail: lattimer@mail.astro.sunysb.edu

\section{Andrew W. Steiner}

Institute for Nuclear Theory, University of Washington,

Seattle, WA 98195, USA

E-mail: steiner3@uw.edu

The supernova remnant Cassiopeia A contains the youngest known neutron star which is also the first one for which real time cooling has ever been observed. In order to explain the rapid cooling of this neutron star, we first present the fundamental properties of neutron stars that control their thermal evolution with emphasis on the neutrino emission processes and neutron/proton superfluidity/superconductivity. Equipped with these results, we present a scenario in which the observed cooling of the neutron star in Cassiopeia $\mathrm{A}$ is interpreted as being due to the recent onset of neutron superfluidity in the core of the star. The manner in which the earlier occurrence of proton superconductivity determines the observed rapidity of this neutron star's cooling is highlighted.

XXXIV edition of the Brazilian Workshop on Nuclear Physics,

5-10 June 2011

Foz de Iguaçu, Parana state, Brasil

\footnotetext{
* Speaker.
} 


\section{Introduction: "neutron stars" do exist}

Neutron stars contain the densest form of cold matter observable in the Universe. Larger energy densities are transiently reached in relativistic heavy ion collisions, but the resulting matter is extremely "hot". Black holes contain a much denser form of matter, but their interior is not observable. Two simple arguments can convince us that such stars, very small and very dense, do exist. First, consider the fastest known radio pulsar, "Ter5ad" (PSR J1748-2446ad) [21], and posit that the observed period of its pulses, $P=1.39 \mathrm{~ms}$, is its rotational period. Using causality, that is, imposing that the velocity at its equator is smaller than the speed of light $c$, one then obtains

$$
v_{\text {equator }}=\Omega R=\frac{2 \pi R}{P}<c \Rightarrow R<\frac{c P}{2 \pi}=65 \mathrm{~km} .
$$

This value of $65 \mathrm{~km}$ for the radius $R$ is only a strict upper limit; detailed theoretical models indicate radii on the order of $10 \mathrm{~km}$. Secondly, assuming that the star is bound by gravity, we can require that the gravitational acceleration $a_{\mathrm{gr}}$ at the equator is larger than the centrifugal acceleration $a_{\mathrm{cf}}$ and obtain

$$
a_{\mathrm{gr}}=\frac{G M}{R^{2}}>a_{\mathrm{cf}}=\Omega^{2} R=\frac{4 \pi^{2} R}{P^{2}} \text { or } \frac{M}{R^{3}}>\frac{4 \pi^{2}}{G P^{2}} \Rightarrow \bar{\rho}=\frac{M}{\frac{4}{3} \pi R^{3}}>8 \times 10^{13} \mathrm{gcm}^{-3} .
$$

Obviously, Newtonian gravity is not accurate in this case, but we can nevertheless conclude that the central density of these stars is comparable, or likely larger, than the nuclear density $\rho_{\text {nuc }} \simeq$ $2.7 \times 10^{14} \mathrm{~g} \mathrm{~cm}^{-3}$. Theoretical models show that densities up to $10 \rho_{\text {nuc }}$ are possibly reachable [26]. In short, a "neutron star" is a gigantic, and compressed, nucleus of the size of a city.

In what follows, we outline the basic properties of neutron stars relevant for describing their thermal evolution with emphasis on the neutrino emission processes and neutron/proton superfluidity/superconductivity. This allows us to present simple analytical models of the cooling of a neutron star in order to gain physical insight. The results of these analytical models are complemented by those of numerical simulations in which full general relativity and the state of the art microphysics are employed. Finally, an interpretation of the observed rapid cooling of the neutron star in Cassiopeia A as due to the recent onset of neutron superfluidity in it core is proffered. The role of proton superconductivity in determining the rapidity of Cas A's cooling is addressed.

\section{Neutron Stars: "pure neutron stars" do not exist}

A "pure neutron star", as originally conceived by Baade \& Zwicky [3] and Oppenheimer \& Volkoff [31] cannot really exist. Neutrons in a ball should decay into protons through

$$
n \rightarrow p+e^{-}+\bar{v}_{e} .
$$

This decay is possible for free neutrons as $m_{n}>m_{p}+m_{e}$. However, given the densities expected within the neutron star interior, the relevant quantities are not the masses, but instead the chemical potentials $\mu_{i}$ ( $i$ denoting the species) of the participants. The matter is degenerate as typical Fermi energies are on the order of tens to hundreds of MeV's, whereas the temperature drops below a few MeV within seconds after the birth of the neutron star in a core collapse supernova [10]. 
Starting with a ball of almost degenerate neutrons, the decay of Eq. (2.1) will generate a Fermi sea of protons, electrons and anti-neutrinos. The interaction mean free paths of anti-neutrinos (and neutrinos) far exceed the size of the star and these can be assumed to leave the star. Thus, the decay will be possible until

$$
\mu_{n}=\mu_{p}+\mu_{e}
$$

where we have neglected $\mu_{v}$. Under this condition, the inverse reaction

$$
p+e^{-} \rightarrow n+v_{e}
$$

also becomes energetically possible. Hence, under equilibrium conditions, which a neutron star will reach within a few tens of seconds after its birth, $\beta$ reactions such as Eq. (2.1) and (2.3) will adjust the chemical composition of matter to that characteristic of $\beta$ (or chemical) -equilibrium determined by Eq. (2.2). A neutron star is not born as a "ball of neutrons" which may decay according to Eq. (2.1), but from the collapse of the iron core of a massive star. It is thus born with an excess of protons so that the reaction Eq. (2.3) initially dominates over Eq. (2.1) in order to reduce the proton fraction and it is only when the neutrinos escape that the final cold $\beta$-equilibrium configuration, Eq. (2.2), is reached.

Notice that once $\mu_{e}>m_{\mu} \simeq 105 \mathrm{MeV}$, muons will appear, and be stably present with $\mu_{\mu}=\mu_{e}$. The condition for the appearance of muons is fulfilled when the density is slightly above $\rho_{\text {nuc }}$. Thus, in all processes we describe below, there will always be the possibility to replace electrons by muons when the density is large enough to allow for their presence.

Let us consider simple expressions for the chemical potentials ${ }^{1}$ :

$$
\begin{aligned}
\mu_{n}=m_{n}+\frac{p_{F}(n)^{2}}{2 m_{n}}+V_{n} & \text { and } & \mu_{p}=m_{p}+\frac{p_{F}(p)^{2}}{2 m_{p}}+V_{p} \\
\mu_{e}=\sqrt{m_{e}^{2} c^{4}+p_{F}(e)^{2} c^{2}}=p_{F}(e) c & \text { and } & \mu_{\mu}=\sqrt{m_{\mu}^{2} c^{4}+p_{F}(\mu)^{2} c^{2}},
\end{aligned}
$$

where $p_{F}(i)$ is the Fermi momentum of species $i$, and $V_{n}$ and $V_{p}$ are the mean-field energies of $n$ and $p$. For the leptons, $V_{e}$ and $V_{\mu}$ are negligibly small, and we have considered that electrons, but not necessarily muons, are ultra-relativistic. With a knowledge of $V_{n}$ and $V_{p}$, the two $\beta$-equilibrium relations Eq. (2.2) and $\mu_{\mu}=\mu_{e}$ can be solved. With four $\mu_{i}$ 's and two equations, a unique solution is obtained by imposing charge neutrality

$$
n_{p}=n_{e}+n_{\mu}
$$

and fixing the baryon density

$$
n_{B}=n_{p}+n_{p} .
$$

With the $n_{i}$ 's and $\mu_{i}$ 's known, one can calculate any thermodynamic potential, in particular the pressure $P$ and energy density $\varepsilon=\rho c^{2}$. Varying the value of $n_{B}$ gives us the equation of state (EOS): $P=P(\rho)$. Given an EOS, an integration of the Tolman-Oppenheimer-Volkoff (TOV) equations of hydrostatic equilibrium provides us with a well defined model of a neutron star.

\footnotetext{
${ }^{1}$ Relativistic expressions for $\mu_{n}$ and $\mu_{p}$ also exist, but are omitted here in the interest of simplicity.
} 


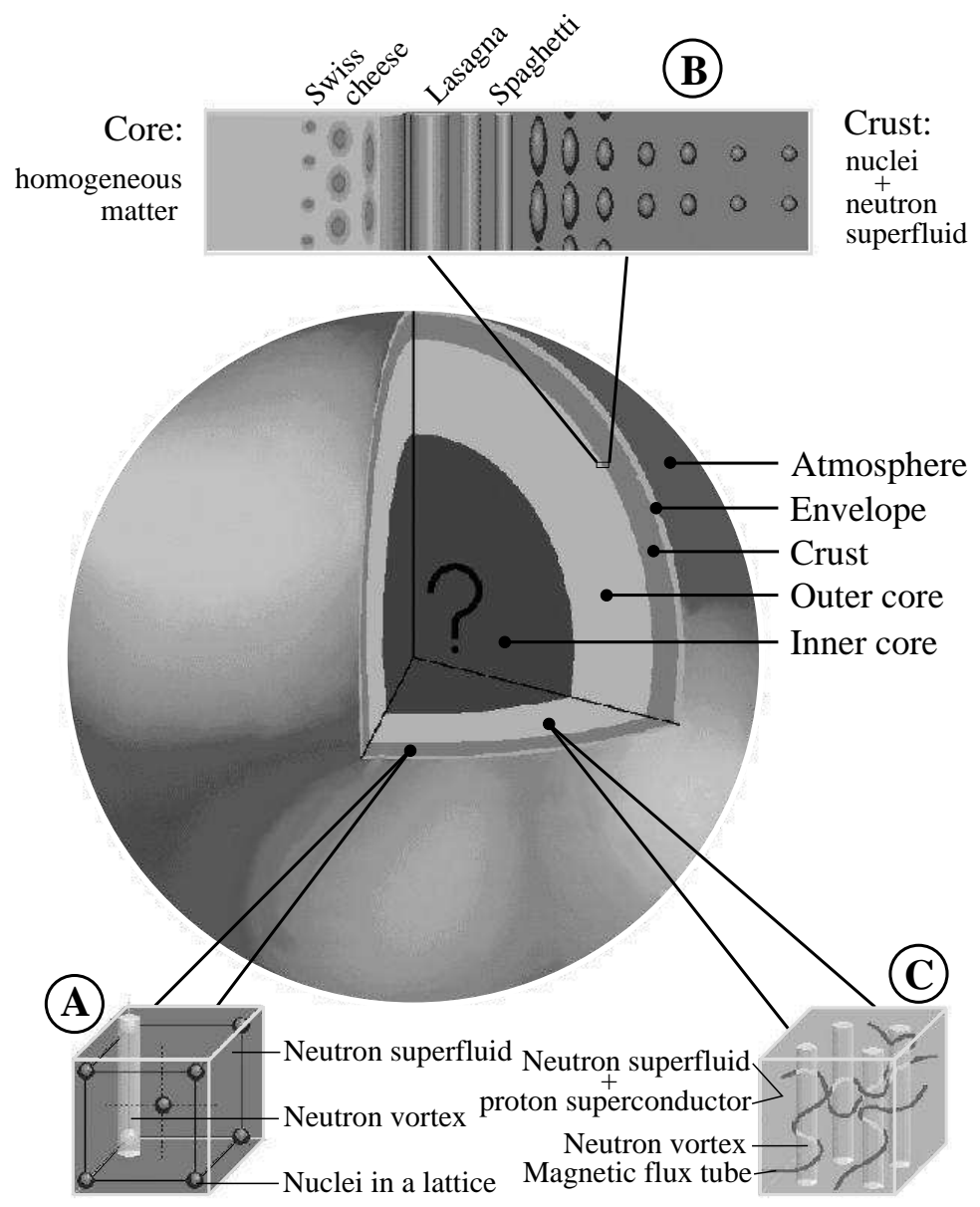

Figure 1: Schematic illustration of the structure of a neutron star; figure taken from [38].

The potentials $V_{n}$ and $V_{p}$ in Eq. (2.4) turn out to be rapidly growing functions of density, and one can anticipate that eventually reactions such as

$$
p+e^{-} \rightarrow \Lambda+\bar{v}_{e} \quad \text { or/and } \quad n+e^{-} \rightarrow \Sigma^{-}+\bar{v}_{e}
$$

may produce hyperons. Hyperons can appear, and be stable, once the corresponding $\beta$-equilibrium conditions are satisfied, i.e., $\mu_{n}=\mu_{\Lambda}$ or/and $\mu_{n}+\mu_{e}=\mu_{\Sigma}^{-}$. At the threshold, where $p_{F}(\Lambda)=0$ or $p_{F}\left(\Sigma^{-}\right)=0$, one can expect that $\left|V_{\Lambda}\right| \ll m_{\Lambda}$ and $\left|V_{\Sigma^{-}}\right| \ll m_{\Sigma^{-}}$and thus $\mu_{\Lambda} \simeq m_{\Lambda}$ and $\mu_{\Sigma^{-}} \simeq m_{\Sigma^{-}}$. Since $m_{\Lambda}$ and $m_{\Sigma^{-}}$are larger than the nucleon mass by only about $200 \mathrm{MeV}$ these hyperons ${ }^{2}$ are good candidates for an "exotic" form of matter in neutron stars. Along similar lines, the lightest mesons, pions and/or kaons, may also appear stably, and can form meson condensates. At even larger densities, the ground state of matter is likely to be one of deconfined quarks. All these possibilities depend crucially on the strong interactions terms, $V_{n}$ and $V_{p}$. As we will not employ them in this chapter, we refer, e.g., to [38] for more details and entries to the original literature.

\footnotetext{
${ }^{2}$ The $\Sigma^{+}$is less favored as its $\beta$-equilibrium condition is $\mu_{\Sigma^{+}}=\mu_{p}=\mu_{n}-\mu_{e}$. Heavier baryon are even less favored, but cannot a priori be excluded.
} 
Figure 1 illustrates our present understanding (or misunderstanding) of the interior of a neutron star, with a black question mark "?" in its densest part. The outer part of the star, its crust, is described briefly in the following subsection.

When only nucleons, plus $e$ 's and $\mu$ 's as implied by charge neutrality and constrained by $\beta$ equilibrium, are considered, the EOS can be calculated with much more confidence than in the presence of "exotic" forms of matter. For illustrative puposes, we will employ the EOS of Akmal, Pandharipande \& Ravenhall [1] ("APR" hereafter) in presenting our results.

Although there is no evidence that any observed "neutron star" or pulsar might actually instead be a pure quark star, theory allows this possibility. Such a star would be nearly completely composed of a mixture of up, down and strange quarks, and would differ from a neutron star in that it would be self-bound rather than held together by gravity.

The reader can find a more detailed presentation and entries to the key literature in [38].

\section{The Neutron Star Crust}

In the outer part of the star, where $\rho<\rho_{\text {nuc }}$, a homogeneous liquid of nucleons is mechanically unstable (spinodal instablity). Stability is, however, restored by the formation of nuclei, or nuclear clusters. At the surface, defined as the layer where $P=0$, we expect the presence of an atmosphere, but there is the possibility of having a solid surface condensed by a sufficiently strong magnetic field [25]. A few meters below the surface, ions are totally ionized by the increasing density (the radius of the first Bohr orbital will be larger than the inter-nuclear distance when $\rho \gtrsim 10^{4} \mathrm{~g} \mathrm{~cm}^{-3}$ ). Matter then consists of a gas/liquid of nuclei immersed in a quantum liquid of electrons. When $\rho \approx 10^{6} \mathrm{~g} \mathrm{~cm}^{-3}, \mu_{e}$ is of the order of $1 \mathrm{MeV}$ and the electrons become relativistic. From here on, the Coulomb correction to the electron gas EOS is negligible and electrons form an almost perfect Fermi gas. However, the Coulomb correction to the ion EOS is not negligible. From a gaseous state at the surface, ions will progressively go through a liquid state and finally crystallize, at densities between $10^{2}$ up to $\sim 10^{10} \mathrm{~g} \mathrm{~cm}^{-3}$ depending on the temperature (within the range of temperatures for which the neutron star is thermally detectable). With growing $\rho$, and the accompanying growth of $\mu_{e}$, it is energetically favorable to absorb electrons into nuclei and, hence, the nuclear species expected to be present have a neutron fraction strongly growing with $\rho$. When $\rho \sim 4-8 \times 10^{11} \mathrm{~g}$ $\mathrm{cm}^{-3}$ (the exact value depending on the assumed chemical composition), one reaches the neutron drip point at which the neutron density is so much larger than that of the proton that not all neutrons are bound to nuclei. Matter then consists of a crystal of nuclei immersed in a perfect Fermi gas of electrons and a quantum liquid of dripped neutrons. This region is usually called the inner crust. In most of this inner crust, the dripped neutrons are predicted to form a superfluid (in a spin-singlet, zero orbital angular momentum, state ${ }^{1} \mathrm{~S}_{0}$ ). All neutron stars we observe are rotating; a superfluid cannot undergo rigid body rotation, but it can simulate it by forming an array of vortices (in the core of which superfluidity is destroyed). (See, e.g., [48].) The resulting structure is illustrated in the inset A of Fig. 1.

At not too high densities, nucleons are correlated at short distances by the strong interaction and anti-correlated at larger distances by the Coulomb repulsion between the nuclei, the former producing spherical nuclei and the latter resulting in the crystallization of the matter. As $\rho$ approaches $\rho_{\text {nuc }}$ the shape of the "nuclei" can undergo drastic changes: the nuclear attraction and 
Coulomb repulsion length-scales become comparable and the system is "frustrated". From spherical shapes, nuclei are expected to become elongated ("spaghettis"), form 2D structures ("lasagnas"), always surrounded by the neutron gas/superfluid which occupies an increasingly small portion of the volume. Then the geometry is inverted, with the dripped neutrons confined into $2 \mathrm{D}$, then $1 \mathrm{D}$ ("anti-spaghettis") and finally 0D ("swiss cheese") bubbles. The homogeneous phase, i.e. the core of the star, is reached when $\rho=\rho_{\mathrm{cc}} \simeq 0.6 \rho_{\text {nuc }}{ }^{3}$. This "pasta" regime is illustrated in the inset B of Fig. 1 and is thought to resemble a liquid crystal [40].

\section{Neutrino Emission Processes}

The thermal evolution of neutron stars with ages $\lesssim 10^{5}$ yrs is driven by neutrino emission. Here we describe the dominant processes. The simplest neutrino emitting processes, Eq. (2.1) and Eq. (2.3), are

$$
\left\{\begin{array}{l}
n \quad \longrightarrow p+e^{-}+\bar{v}_{e} \\
p+e^{-} \longrightarrow n+v_{e},
\end{array}\right.
$$

and are generally referred to as the direct Urca ("DU") cycle.

By the condition of $\beta$-equilibrium the cycle naturally satisfies energy conservation, but momentum conservation is much more delicate. Due to the high degeneracy, all participating particles have momenta $p(i)$ equal (within a small $T \ll T_{F}$ correction) to their Fermi momenta $p_{F}(i)$. As $p_{F}(i) \propto n_{i}^{1 / 3}$ and $n_{p} \sim n_{e} \ll n_{n}$, momentum conservation is not a priori guaranteed. It is easy to see that, in the absence of muons and hence with $n_{p}=n_{e}$, the "triangle rule" for momentum conservation requires that $x_{p}>1 / 9 \simeq 11 \%$, whereas at $\rho \sim \rho_{\text {nuc }}$ we have $x_{p} \simeq 5 \%$. In the presence of muons, which appear just above $\rho_{\text {nuc }}$, the condition is stronger and one needs $x_{p}$ larger than about $15 \%$ [27]. The proton fraction $x_{p}$ grows with density, its growth being directly determined by the growth of the nuclear symmetry energy, so that the critical proton fraction for the DU process is likely reached at some supra-nuclear density [27]. For the EOS of APR, the corresponding critical neutron star mass for the allowance of the DU process is $1.97 \mathrm{M}_{\odot}$, but other EOS's predict smaller values.

At densities below the threshold density for the DU process, a variant of this process, the modified Urca (MU) process

$$
\left\{\begin{array}{l}
n+n^{\prime} \longrightarrow p+n^{\prime}+e^{-}+\bar{v}_{e} \\
p+n^{\prime}+e^{-} \longrightarrow n+n^{\prime}+v_{e}
\end{array}\right.
$$

can operate as advantage is taken of neighboring particles in the medium [17]. A bystander neutron $n^{\prime}$ can take or give the needed momentum for momentum conservation. The processes in Eq. (3.2) show the neutron branch of the MU process and in the proton branch $n^{\prime}$ is replaced by a proton $p^{\prime}$. As it involves the participation of five degenerate particles, the MU process is much less efficient than the DU process. Unlike the DU processes which require sufficient amount of protons, both branches of the MU process operate at any density when neutrons and protons are present.

\footnotetext{
${ }^{3} \rho_{\text {nuc }}$ correspond to the density of symmetric nuclear matter, i.e. with a proton fraction $x_{p}=50 \%$, and zero pressure, whereas in a neutron star crust at $\rho \sim \rho_{\text {nuc }}$ one has $x_{p} \simeq 3-5 \%$.
} 


\begin{tabular}{|c|c|c|c|}
\hline Name & Process & $\begin{array}{c}\text { Emissivity } \\
\left(\mathrm{erg} \mathrm{cm}^{-3} \mathrm{~s}^{-1}\right)\end{array}$ & \\
\hline $\begin{array}{l}\text { Modified Urca } \\
\text { (neutron branch) }\end{array}$ & $\mid \begin{array}{l}n+n \rightarrow n+p+e^{-}+\bar{v}_{e} \\
n+p+e^{-} \rightarrow n+n+v_{e}\end{array}$ & $\sim 2 \times 10^{21} R T_{9}^{8}$ & Slow \\
\hline $\begin{array}{l}\text { Modified Urca } \\
\text { (proton branch) }\end{array}$ & 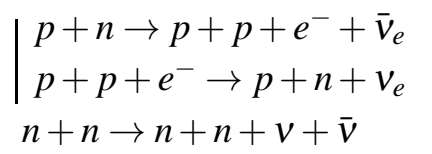 & $\sim 10^{21} R T_{9}^{8}$ & Slow \\
\hline Bremsstrahlungs & $\begin{array}{l}n+p \rightarrow n+p+v+\bar{v} \\
p+p \rightarrow p+p+v+\bar{v}\end{array}$ & $\sim 10^{19} R T_{9}^{8}$ & Slow \\
\hline Cooper pair & $\begin{array}{l}n+n \rightarrow[n n]+v+\bar{v} \\
p+p \rightarrow[p p]+v+\bar{v}\end{array}$ & $\begin{array}{l}\sim 5 \times 10^{21} R T_{9}^{7} \\
\sim 5 \times 10^{19} R T_{9}^{7}\end{array}$ & Medium \\
\hline $\begin{array}{l}\text { Direct Urca } \\
\text { (nucleons) }\end{array}$ & $\mid \begin{array}{l}n \rightarrow p+e^{-}+\bar{v}_{e} \\
p+e^{-} \rightarrow n+v_{e}\end{array}$ & $\sim 10^{27} R T_{9}^{6}$ & Fast \\
\hline $\begin{array}{l}\text { Direct Urca } \\
(\Lambda \text { hyperons })\end{array}$ & $\mid \begin{array}{l}\Lambda \rightarrow p+e^{-}+\bar{v}_{e} \\
p+e^{-} \rightarrow \Lambda+v_{e}\end{array}$ & $\sim 10^{27} R T_{9}^{6}$ & Fast \\
\hline $\begin{array}{l}\text { Direct Urca } \\
\left(\Sigma^{-} \text {hyperons }\right)\end{array}$ & $\mid \begin{array}{l}\Sigma^{-} \rightarrow n+e^{-}+\bar{v}_{e} \\
n+e^{-} \rightarrow \Sigma^{-}+v_{e}\end{array}$ & $\sim 10^{27} R T_{9}^{6}$ & Fast \\
\hline$\pi^{-}$condensate & $n+<\pi^{-}>\rightarrow n+e^{-}+\bar{v}_{e}$ & $\sim 10^{26} R T_{9}^{6}$ & Fast \\
\hline$K^{-}$condensate & $n+<K^{-}>\rightarrow n+e^{-}+\bar{v}_{e}$ & $\sim 10^{25} R T_{9}^{6}$ & Fast \\
\hline $\begin{array}{l}\text { Direct Urca cycle } \\
\text { (u-d quarks) }\end{array}$ & $\mid \begin{array}{l}d \rightarrow u+e^{-}+\bar{v}_{e} \\
u+e^{-} \rightarrow d+v_{e}\end{array}$ & $\sim 10^{27} R T_{9}^{6}$ & Fast \\
\hline $\begin{array}{l}\text { Direct Urca cycle } \\
\text { (u-s quarks) }\end{array}$ & $\mid \begin{array}{l}s \rightarrow u+e^{-}+\bar{v}_{e} \\
u+e^{-} \rightarrow s+v_{e}\end{array}$ & $\sim 10^{27} R T_{9}^{6}$ & Fast \\
\hline
\end{tabular}

Table 1: A sample of neutrino emission processes. $T_{9}$ is temperature $T$ in units of $10^{9} \mathrm{~K}$ and the $R$ 's are control factors to include the suppressing effects of pairing (see §5).

In the presence of hyperons, DU and MU processes which are obvious generalizations of the nucleon-only process can also occur [42]. When they appear, the $\Lambda$ 's have a density much smaller than that of the neutron and hence a smaller Fermi momentum. Consequently, momentum conservation in the DU cycle $p+e^{-} \rightarrow \Lambda+\bar{v}_{e}$ and $\Lambda \rightarrow p+e^{-}+v_{e}$ is easily satisfied, requiring an $x_{\Lambda}$ of only $\sim 3 \%$. Notice that if the nucleon DU process is kinematically forbidden, the hyperon DU process $\Sigma^{-} \rightarrow n+e^{-}+\bar{v}_{e}$ with $n+e^{-} \rightarrow \Sigma^{-}+v_{e}$ is also kinematically forbidden, whereas the no-nucleon DU process $\Sigma^{-} \rightarrow \Lambda+e^{-}+\bar{v}_{e}$ together with $\Lambda+e^{-} \rightarrow \Sigma^{-}+v_{e}$ may be possible and require very low threshold fractions $x_{\Lambda}$ and $x_{\Sigma}^{-}$.

In deconfined quark matter, DU processes such as $u+e \rightarrow d+\bar{v}_{e}$ and $d \rightarrow u+e+v_{e}$ are possible [23]. Reactions in which the $d$ quark is replaced by an $s$ quark can also occur in the case $s$ quarks are present.

In the presence of a meson condensate copious neutrino emission in processes such as

$$
\left\{\begin{array}{l}
n+e^{-} \longrightarrow n+\pi^{-}+v_{e} \\
n+\pi^{-} \longrightarrow n+e^{-}+\bar{v}_{e}
\end{array}\right.
$$


and

$$
\left\{\begin{array}{l}
n+e^{-} \longrightarrow n+K^{-}+v_{e} \\
n+K^{-} \longrightarrow n+e^{-}+\bar{v}_{e}
\end{array}\right.
$$

occur $[29,9]$. As the meson condensate is a macroscopic object there is no restriction arising from momentum conservation in these processes.

Finally, another class of processes, bremsstrahlung, is possible through neutral currents [16]. Reactions such as

$$
\begin{gathered}
n+n^{\prime} \longrightarrow n+n^{\prime}+v_{e}+\bar{v}_{e} \\
p+p^{\prime} \longrightarrow p+p^{\prime}+v_{e}+\bar{v}_{e} \\
n+p^{\prime} \longrightarrow n+p^{\prime}+v_{e}+\bar{v}_{e}
\end{gathered}
$$

are less efficient, by about 2 orders of magnitude, than the MU processes, but may make some contribution in the case that the MU process is suppressed by pairing of neutrons or protons as we will see in $§ 5$.

In Table 1 we list these processes with order of magnitude estimates of their neutrino emissivities. Most noticeable is the clear distinction between processes involving 5 degenerate fermions with a $T^{8}$ dependence, which are labeled as "slow", and those with only 3 degenerate fermions with a $T^{6}$ dependence, which are several orders of magnitude more efficient and labeled as "fast". The difference in the $T$ dependence is important and simply related to phase space arguments which are outlined below. The Cooper pair process $[15,51]$ will be described in $\S 5$.

The reader can find a detailed description of neutrino emission processes in [54] and an alternative point of view in [50].

\section{Temperature dependence of neutrino emission}

We turn now to briefly describe how the specific temperature dependence of the neutrino processes described above emerges. Consider first the simple case of the neutron beta decay. The weak interaction is described by the Hamiltonian $\mathscr{H}_{I}=\left(G_{F} / \sqrt{2}\right) B_{\mu} L^{\mu}$, where $G_{F}$ is Fermi's constant, and $L^{\mu}=\bar{\psi}_{e} \gamma^{\mu}\left(1-\gamma_{5}\right) \psi_{v}$ and $B_{\mu}=\bar{\psi}_{p} \gamma_{\mu}\left(C_{V} 1-C_{A} \gamma_{5}\right) \psi_{n}$ are the lepton and baryon currents, respectively. In the non relativistic approximation, one has $B^{0}=\cos \theta_{c} \Psi_{p}^{\dagger} \Psi_{n}$ and $B^{i}=$ $-\cos \theta_{c} g_{A} \Psi_{p}^{\dagger} \sigma^{i} \Psi_{n}$ where $\theta_{c}$ is the Cabibbo angle and $g_{A}$ the axial-vector coupling. Fermi's Golden rule gives us for the neutron decay rate

$$
W_{i \rightarrow f}=\iiint \frac{d^{3} p_{v}}{(2 \pi)^{3}} \frac{d^{3} p_{e}}{(2 \pi)^{3}} \frac{d^{3} p_{p}}{(2 \pi)^{3}}(2 \pi)^{4} \delta^{4}\left(P_{f}-P_{i}\right) \cdot\left|M_{f i}\right|^{2},
$$

i.e., a sum of $(2 \pi)^{4} \delta^{4}\left(P_{f}-P_{i}\right) \cdot\left|M_{f i}\right|^{2}$ over the phase space of all final states $f=\left(\vec{p}_{\bar{v}}, \vec{p}_{e}, \vec{p}_{p}\right)$. The integration gives the well known result: $W_{\beta}=\frac{1}{2 \pi^{3}}\left[G_{F}^{2} \cos ^{2} \theta_{c}\left(1+3 g_{A}^{2}\right) m_{e}^{5} c^{4}\right] w_{\beta}$, where $w_{\beta} \sim 1$ takes into account small Coulomb corrections. This gives us the neutron mean life, $\tau_{n} \simeq 15 \mathrm{~min}$., or, measuring $\tau_{n}$, a measurement of $G_{F}$ (modulo $\cos \theta_{c}$ and $w_{\beta}$ ).

The emissivity $\varepsilon^{\mathrm{DU}}$ of the DU process (the Feynman diagram for this process is shown in Fig. 2) can be obtained by the same method as above leading to

$$
\varepsilon^{\mathrm{DU}}=\iiint \int \frac{d^{3} p_{\bar{v}}}{(2 \pi)^{3}} \frac{d^{3} p_{e}}{(2 \pi)^{3}} \frac{d^{3} p_{p}}{(2 \pi)^{3}} \frac{d^{3} p_{n}}{(2 \pi)^{3}}\left(1-f_{e}\right)\left(1-f_{p}\right) f_{n} \cdot(2 \pi)^{4} \delta^{4}\left(P_{f}-P_{i}\right)\left|M_{f i}\right|^{2} \cdot E_{v}
$$




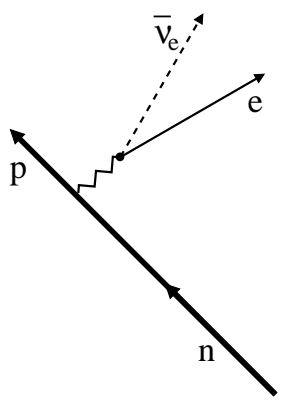

Direct Urca

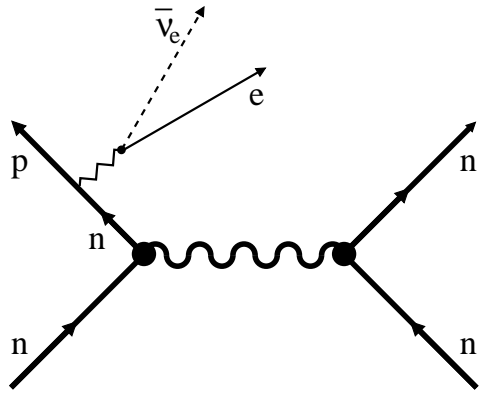

Modified Urca

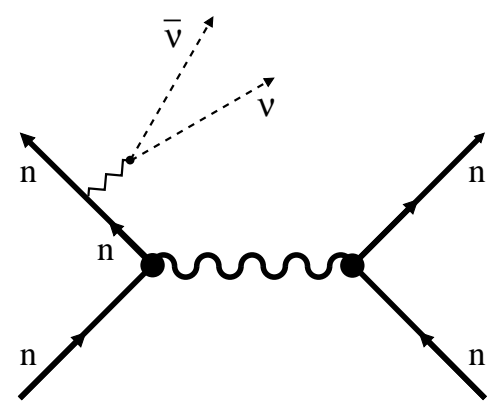

Bremsstrahlung

Figure 2: Feynman diagrams for the indicated neutrino emitting processes.

with an extra factor $E_{v}$ for the neutrino energy and the phase space sum now includes the initial $n$. The $f_{i}$ terms, $f_{i}$ being the Fermi-Dirac distribution for particle $i$ at temperature $T$, take into account: (1) the probability to have a $n$ in the initial state, $f_{n}$, and (2) the probabilities to have available states for the final $e$ and $p$, denoted by $\left(1-f_{e}\right)$ and $\left(1-f_{p}\right)$, respectively. We do not introduce a Pauli blocking factor $\left(1-f_{\bar{v}}\right)$ for the anti-neutrino as it is assumed to be able to freely leave the star (i.e., $f_{\bar{v}}=0$ ). When performing the phase space integrals, each degenerate fermion gives us a factor $T$, as particles are restricted to be within a shell of thickness $k_{B} T$ of their respective Fermi surfaces. The anti-neutrino phase space gives a factor $T^{3}$. The factors $E_{v} \sim T$ and the delta function $\delta^{4}\left(P_{f}-P_{i}\right)$ gives a factor $T^{-1}$ (it cancels one of the $T$ 's from the degenerate fermions). Altogether, we find that

$$
\varepsilon^{\mathrm{DU}} \propto T^{3} \cdot T \cdot T \cdot T \cdot \frac{1}{T} \cdot(1)^{2} \cdot T=T^{6},
$$

where the $(1)^{2}$ factor emphasizes that the squared matrix element $\left|M_{f i}\right|^{2}$ is T-independent. An explicit expression for the neutrino emissivity for the DU process can be found in [27] .

Figure Fig. 2 shows a Feynman diagram for the MU process. There are two more such diagrams in which the weak interaction vertex is attached to one of the two incoming legs. In this case, the $T$-power counting gives

$$
\varepsilon^{\mathrm{MU}} \propto T^{3} \cdot T \cdot T \cdot T \cdot T \cdot T \cdot \frac{1}{T} \cdot(1)^{2} \cdot T=T^{8}
$$

where the $\left|M_{f i}\right|^{2}$ now involves a strong interaction vertex, the wavy line in Fig. 2, but is still $T$ independent. Notice that in the MU case, the internal neutron is off-shell by an amount $\simeq \mu_{e}$ which does not introduce any extra $T$-dependence as we are working in the case $E_{F}(e) \gtrsim 100 \mathrm{MeV} \gg T$. Reference [17] contains the expression from which neutrino emissivity from the MU process can be calculated. Considering finally the $n-n$ bremsstrahlung process. One diagram is shown in Fig. 2 and there are three more diagrams with the weak interaction vertices attached to the other three external lines. The $T$-power counting now gives

$$
\varepsilon^{\mathrm{Br}} \propto T^{3} \cdot T^{3} \cdot T \cdot T \cdot T \cdot T \cdot \frac{1}{T} \cdot\left(\frac{1}{T}\right)^{2} \cdot T=T^{8}
$$


with two $T^{3}$ factors for the neutrino pair and a $\left(T^{-1}\right)^{2}$ from the matrix element: the intermediate neutron is almost on-shell, with an energy deficit $\sim T$, and its propagator gives us a $T^{-1}$ dependence for $M_{f i}$. A working expression for the bremsstrahlung process can be found in [17].

\section{Neutron Star Cooling}

The basic features of the cooling of a neutron star are best illustrated by considering the energy balance equation for the star in its Newtonian formulation ${ }^{4}$ :

$$
\frac{d E_{\mathrm{th}}}{d t}=C_{\mathrm{v}} \frac{d T}{d t}=-L_{v}-L_{\gamma}+H,
$$

where $E_{\mathrm{th}}$ is the thermal energy content of the star, $T$ its internal temperature, and $C_{\mathrm{v}}$ its total specific heat. The two energy sinks are neutrino luminosity $L_{v}$, described in $\$ 3$, and the surface photon luminosity $L_{\gamma}$, discussed in $\S 4.2$. The source term $H$ would include heating mechanisms as, e.g., magnetic field decay, which we will not consider here.

\subsection{Specific Heat}

The dominant contributions to the specific heat $C_{\mathrm{v}}$ come from the core, constituting more than $90 \%$ of the total volume, whose constituents are quantum liquids of leptons, baryons, mesons, and possibly deconfined quarks at the highest densities. Hence one has

$$
C_{\mathrm{V}}=\sum_{i} C_{\mathrm{V}, i} \quad \text { with } \quad C_{\mathrm{V}, i}=\iiint c_{\mathrm{v}, i} d \mathrm{v} \quad(i=\mathrm{e}, \mu, \mathrm{n}, \mathrm{p}, \text { hyperons, quarks, }, \ldots),
$$

where $c_{\mathrm{v}, i}$ is the specific heat, per unit volume, of component $i$. For normal (i.e., unpaired) degenerate fermions, the standard Fermi liquid result [6]

$$
c_{\mathrm{v} i}=N(0) \frac{\pi^{2}}{3} k_{B}^{2} T \text { with } N(0)=\frac{m_{i}^{*} p_{F i}}{\pi^{2} \hbar^{3}}
$$

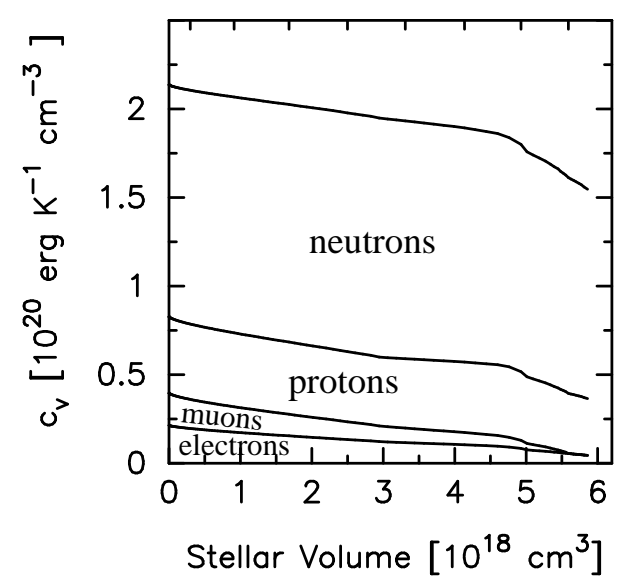

Figure 3: Cumulative specific heats of e, $\mu, \mathrm{p}$, and $\mathrm{n}$ vs. stellar volume in the core of a $1.4 M_{\odot}$ star built using the EOS of APR, at temperature $T=10^{9} \mathrm{~K}$. Nucleons are assumed to be unpaired. No hyperons or quarks are present in the EOS. This figure is adapted from [35].

\footnotetext{
${ }^{4}$ Numerical results to be shown later include full general relativistic effects.
} 
can be used, where $m^{*}$ is the fermion's effective mass. In Fig. 3, the various contributions to $C_{V}$ are illustrated. When baryons, and quarks, become paired, as briefly described in $\$ 5$, their contribution to $C_{\mathrm{v}}$ is strongly suppressed at temperatures $T \ll T_{\mathrm{c}}$ ( $T_{\mathrm{c}}$ being the corresponding critical temperature). Extensive baryon, and quark, pairing can thus significantly reduce $C_{\mathrm{v}}$, but by at most a factor of order ten as the leptons do not pair. The crustal contribution is in principle dominated by neutrons in the inner crust but, as these are certainly extensively paired, practically only the nuclear lattice and electrons contribute.

\subsection{Photon emission and the neutron star envelope}

Thermal photons from the neutron star surface are emitted at the photosphere, which is usually in an atmosphere but could be a solid surface in the presence of a very strong magnetic field. The atmosphere, which is only a few centimeters thick, presents a temperature gradient. Consequently, photons of increasing energy escape from deeper and hotter layers. It is customary to define an effective temperature, $T_{e}$, so that the total surface photon luminosity, by analogy with the blackbody emission, is written as

$$
L_{\gamma}=4 \pi R^{2} \sigma_{\mathrm{SB}} T_{e}^{4},
$$

where $\sigma_{\mathrm{SB}}$ is the Stefan-Boltzmann constant. Observationally, $L_{\gamma}$ and $T_{e}$ are red-shifted and Eq. (4.4) is rewritten as

$$
L_{\gamma \infty}=4 \pi R_{\infty}^{2} \sigma_{\mathrm{SB}} T_{e \infty}^{4}
$$

where $L_{\gamma_{\infty}}=\mathrm{e}^{2 \phi} L_{\gamma}, T_{e \infty}=\mathrm{e}^{\phi} T_{e}$, and $R_{\infty}=\mathrm{e}^{-\phi} R$. Here $\mathrm{e}^{-\phi}=1+z$, with $z$ being the red-shift, and $\mathrm{e}^{2 \phi}$ is the $g_{00}$ coefficient of the Schwarzschild metric, i.e.,

$$
\mathrm{e}^{\phi} \equiv \sqrt{1-\frac{2 G M}{R c^{2}}}
$$

Notice that $R_{\infty}$ has the physical interpretation of being the star's radius that an observer would measure trigonometrically, if this were possible.

In a detailed cooling calculation, the time evolution of the whole temperature profile in the star is followed. However, the uppermost layers have a thermal time-scale much shorter than the interior of the star and are practically always in a steady state. It is hence common to treat these layers separately as an envelope. Encompassing a density range from $\rho_{b}$ at its bottom (typically $\rho_{b}=10^{10}$ $\mathrm{g} \mathrm{cm}^{-3}$ ) up to that at the photosphere, and a temperature range from $T_{b}$ to $T_{e}$, the envelope is about one hundred meters thick. Due to the high thermal conductivity of degenerate matter, stars older than a few decades have an almost uniform internal temperature except within the envelope which acts as a thermal blanket insulating the hot interior from the colder surface. A simple relationship between $T_{b}$ and $T_{e}$ can be formulated as in [19]:

$$
T_{e} \simeq 10^{6}\left(\frac{T_{b}}{10^{8} \mathrm{~K}}\right)^{0.5+\alpha}
$$

with $\alpha \ll 1$. The precise $T_{e}-T_{b}$ relationship depends on the chemical composition of the envelope. The presence of light elements, resulting in larger thermal conductivities, implies a larger $T_{e}$ for the same $T_{b}$ compared to the case of a heavy element envelope. Magnetic fields also alter this $T_{e}-T_{b}$ relationship (see, e.g., [34] for more details). 


\subsection{Some simple analytical solutions}

As the essential ingredients entering Eq. (4.1) can all be approximated by power-law functions, one can obtain simple and illustrative analytical solutions. Let us write

$$
C_{\mathrm{V}}=C_{9} \cdot T_{9}, \quad L_{v}=N_{9} \cdot T_{9}^{8} \quad \text { and } \quad L_{\gamma}=S_{9} \cdot T_{9}^{2+4 \alpha},
$$

where $T_{9} \equiv T /\left(10^{9} \mathrm{~K}\right)$. As written, $L_{v}$ considers slow neutrino emission involving five degenerate fermions from the modified Urca and the similar bremsstrahlung processes, summarized in Table 1. The photon luminosity $L_{\gamma}$ is obtained from Eq. (4.4) using the simple expression in Eq. (4.7). Typical values are $C_{9} \simeq 10^{39} \mathrm{erg} \mathrm{K}^{-1}, N_{9} \simeq 10^{40} \mathrm{erg} \mathrm{s}^{-1}$ and $S_{9} \simeq 10^{33} \mathrm{erg} \mathrm{s}^{-1}$ (see Table 3 in [34] for more details). In young stars neutrinos dominate the energy losses (in the so-called neutrino cooling era) and photons take over after about $10^{5}$ yrs (in the photon cooling era).

Neutrino cooling era: In this case, we can neglect $L_{\gamma}$ in Eq. (4.1) and find

$$
t=\frac{10^{9} C_{9}}{6 N_{9}}\left(\frac{1}{T_{9}^{6}}-\frac{1}{T_{09}^{6}}\right) \quad \longrightarrow \quad T=10^{9} \mathrm{~K}\left(\tau_{\mathrm{MU}} / t\right)^{1 / 6} \quad\left(\text { when } T \ll T_{0}\right)
$$

with a MU cooling time-scale $\tau_{\mathrm{MU}}=19^{9} C_{9} / 6 N_{9} \sim 1 \mathrm{yr}$ when the star reaches the asymptotic solution ( $T \ll T_{0}, T_{0}$ being the initial temperature at time $t=t_{0}=0$ ).

Photon cooling era: In this era, $L_{v}$ becomes negligible compared to $L_{\gamma}$ so that we obtain

$$
t=t_{1}+\frac{C_{9}}{4 \alpha S_{9} 10^{9+36 \alpha}}\left(\frac{1}{T_{9}^{4 \alpha}}-\frac{1}{T_{19}^{4 \alpha}}\right) \quad \longrightarrow \quad T \propto t^{-\frac{1}{4 \alpha}} \quad\left(\text { when } T \ll T_{1}\right)
$$

where $T_{1}$ is $T$ at time $t_{1}$. Notice here that the slope, $-1 / 4 \alpha$, of the asymptotic solution is strongly affected by any small change in the envelope structure, Eq. (4.7).

\subsection{Some numerical solutions}

Numerical simulations of a cooling neutron star use an evolution code in which the energy balance and energy transport equations in their fully general relativistic forms are solved, usually assuming spherical symmetry and with a numerical radial grid of several hundred zones ${ }^{5}$. A set of cooling curves that illustrate the difference between cooling driven by the modified Urca and the direct Urca processes is presented in Fig. 4. Cooling curves of eight different stars of increasing mass are shown, using an equation of state model, from [41], which allows the DU process at densities above $1.25 \times 10^{15} \mathrm{~g} \mathrm{~cm}^{-3}$, i.e., above a critical neutron star mass of $1.35 M_{\odot}$. Notice that the equation of state used is a parametric one and its parameters were specifically adjusted to obtain a critical mass of $1.35 M_{\odot}$ which falls within the expected range of isolated neutron star masses; other equations of state can result in very different critical masses. The difference arising from slow and fast neutrino processes is clear.

Four successive cooling stages are marked in the figure. The neutrino cooling era is marked stage 3 and the photon cooling era is marked stage 4 . As the figure shows $T_{e}$, and since $T_{e} \sim$ $T^{0.5}$, the slopes of the cooling curves in this figure are rescaled from the values of Eq. (4.9) and (4.10), i.e., they become $\approx-1 / 12$ and $\approx-1 / 8 \alpha$, respectively. The simple analytical model of the

\footnotetext{
${ }^{5}$ Such a code, NSCool, is available at: http: / / www . astroscu. unam.mx/neutrones/NSCool/
} 


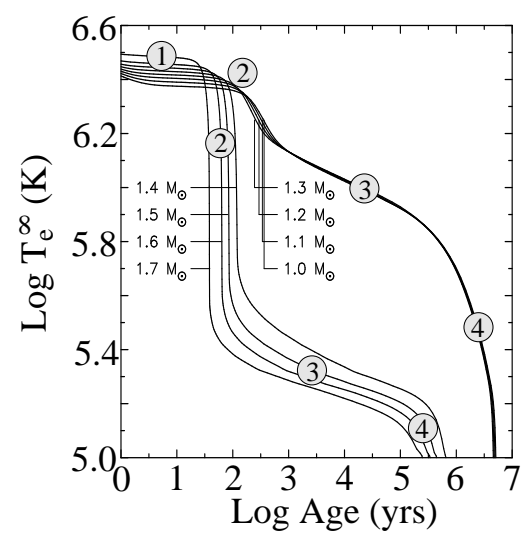

Figure 4: Slow versus fast cooling (see text for details of stages 1 through 4 marked in the figure). Figure adapted from [33].

previous section considered a single temperature in the stellar interior. For a young star, whose age is smaller that its thermal relaxation time, the interior temperature has a very complicated radial profile. In particular, the core cools much faster than the crust, due to its much stronger neutrino emission. During stage 1 in Fig. 4, the surface temperature is controlled by processes occurring in the outer layers of the crust and is totally independent of the temperature deeper in the star. (One can appreciate that the more massive stars have a larger $T_{e}$ at that time; this is mostly due to the fact that their blanketing envelopes are thinner than in low mass stars.) The rapid drop in $T_{e}$ occurring during stage 2 corresponds to the thermal relaxation of the crust; the star's age becomes comparable to the heat transport time-scale from the crust to the core and the crustal heat flows into the core. After stage 2, the stellar interior is essentially isothermal with a strong temperature gradient present only in the envelope and the simple analytical solutions presented above apply.

\section{Pairing and its Effects}

Pairing, which induces superfluidity in the case of degenerate neutral fermions and superconductivity for charged fermions, is expected to occur between neutrons/protons in the interior of neutron stars [30]. The Cooper Theorem [12] states that, in a system of degenerate fermions the Fermi surface is unstable, at $T=0$, due to the formation of Cooper pairs if there is an attractive interaction in some spin-angular momentum channel. The essence of the BCS theory [5] is that as a result of this instability there is a collective reorganization of the particles at energies around the Fermi energy and the appearance of a gap in the quasi-particle spectrum (see Fig. 5) which is the binding energy of a Cooper pair. At high enough temperature the gap $\Delta(T)$ vanishes and the system is in the normal state. The transition to the superfluid/superconducting state is a second order phase transition and the gap $\Delta(T)$ is its order parameter (see Fig. 6). Explicitly, $\Delta(T)=0$ when $T>T_{c}$ and, when $T$ drops below $T_{c}, \Delta(T)$ grows rapidly but continuously, with a discontinuity in its slope at $T=T_{c}$. This is in sharp contrast with a first order phase transition, in which the transition occurs entirely at $T_{c}$ (see left panel of Fig. 6) and will be of paramount importance for our purpose. In the BCS theory, which remains approximately valid for nucleons, the relationship between the gap and 
Normal Fermi Liquid

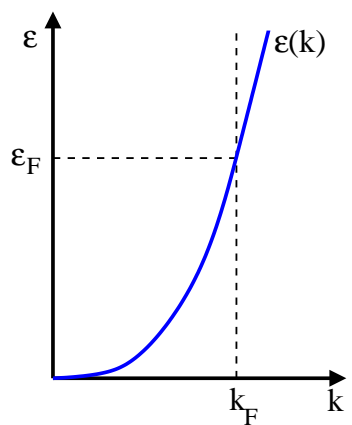

Superfluid Fermions

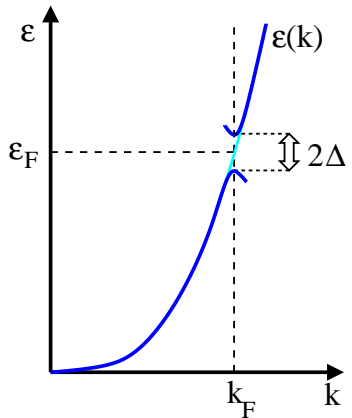

Figure 5: Comparison of quasi-particle spectra, $\varepsilon(k)$, i.e., the pole in the 2-point Green's function, for a normal and a superfluid Fermi liquid. The reorganization of particles at $\varepsilon \sim \varepsilon_{F}$ into Cooper pairs results in the development a gap $2 \Delta(k)$ in the spectrum so that no particle can have an energy between $\varepsilon_{F}-\Delta\left(k_{F}\right)$ and $\varepsilon_{F}+\Delta\left(k_{F}\right)$.

$T_{c}$ is

$$
\Delta(T=0) \simeq 1.75 k_{B} T_{c}
$$

In a normal Fermi system at $T=0$, all particles are in states with energies $\varepsilon \leq \varepsilon_{F}$. When $T>0$, states with energies $\varepsilon \gtrsim \varepsilon_{F}$ can be smoothly occupied (left panel of Fig. 5) resulting in a smearing of the particle distribution around $\varepsilon_{F}$ in a range $\sim k_{B} T$. It is precisely this smooth smearing of energies around $\varepsilon_{F}$ which produces the linear $T$ dependence of $c_{v}, \S 4.1$, and the $T^{6}$ or $T^{8}$ dependence of the neutrino emissivities, $\S 3$.

In a superfluid/superconducting Fermi system at $T=0$, all particles are in states with energies $\varepsilon \leq \varepsilon_{F}$ (actually, $\varepsilon \leq \varepsilon_{F}-\Delta$ ). When $0<T<T_{c}$, states with energy $\varepsilon>\varepsilon_{F}$ (actually, $\varepsilon \geq \varepsilon_{F}+\Delta$ ) can be populated. However, in contrast to the smooth filling of levels above $\varepsilon_{F}$ in the case of a

First Order Phase Transition

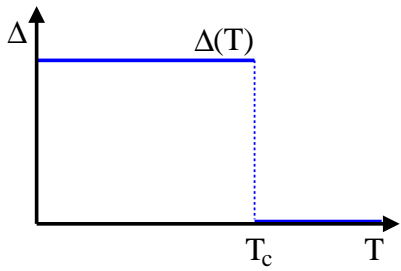

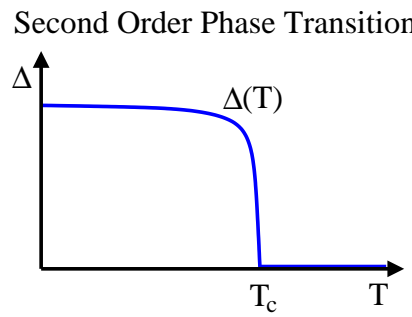

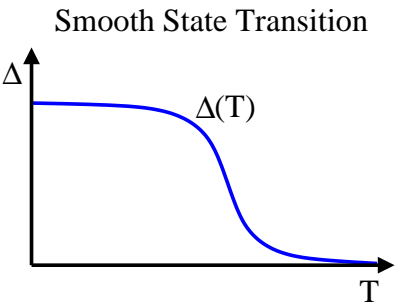

Figure 6: Temperature evolution of the state of a system parametrized by an "order" parameter, $\Delta(T)$. First order phase transition: discontinuous change of $\Delta$ at $T_{c}$; latent heat due to the entropy difference between the two states. (Examples: solid $\leftrightarrow$ liquid; liquid $\leftrightarrow$ gas below the critical point.)

Second order phase transition: continuous change of $\Delta$ but with a discontinuity in the slope at $T_{c}$; no latent heat but a discontinuity in specific heat. (Examples: superfluid $\leftrightarrow$ normal fluid; ferromagnetic $\leftrightarrow$ paramagnetic.)

Smooth state transition: continuous change of $\Delta$ with no critical temperature; increase of specific heat in the regime where $\Delta(T)$ changes rapidly. (Examples: liquid $\leftrightarrow$ gas above the critical point; atomic gas $\leftrightarrow$ plasma.) 

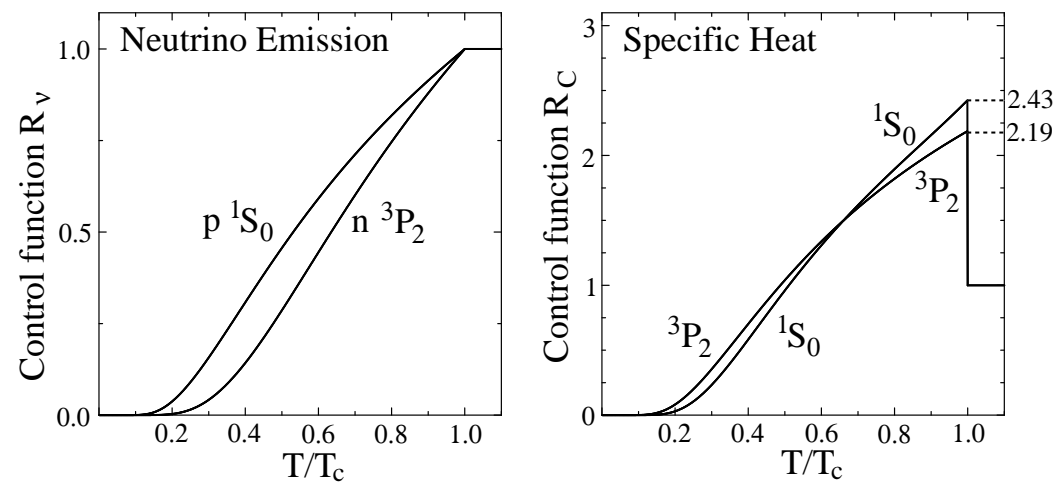

Figure 7: Control functions for MU neutrino emission (left panel) and specific heat (right panel).

normal Fermi liquid, the presence of the $2 \Delta(T)$ gap in the spectrum implies that the occupation probability is strongly suppressed by a Boltzmann factor $\sim \exp \left[-2 \Delta(T) / k_{B} T\right]$. Consequently, all physical properties/processes depending on thermally excited particles, such as the specific heat and the neutrino emission processes described in $\S 3$, are strongly suppressed when $T \ll T_{c}$. In practice, for numerical simulations of neutron star cooling, these suppressions are implemented through "control functions" $R_{*}$ such that

$$
\begin{aligned}
c_{\mathrm{v}} \rightarrow c_{\mathrm{v}}^{\text {Paired }} & =R_{c} c_{\mathrm{v}}^{\text {Normal }} \\
\varepsilon_{v} \rightarrow \varepsilon_{v}^{\text {Paired }} & =R_{v} \varepsilon_{v}^{\text {Normal }} .
\end{aligned}
$$

There is a large family of such functions, for each process and they moreover depend on how many of the participating particles are paired and on the specific kind of pairing; a few examples are displayed in Fig. 7 [54].

\subsection{Theoretical expectations on pairing gaps}

Soon after the development of the BCS theory, Bohr, Mottelson \& Pine [8] pointed out that excitation energies of nuclei show a gap, as shown in the left panel of Fig. 8. Even-even nuclei
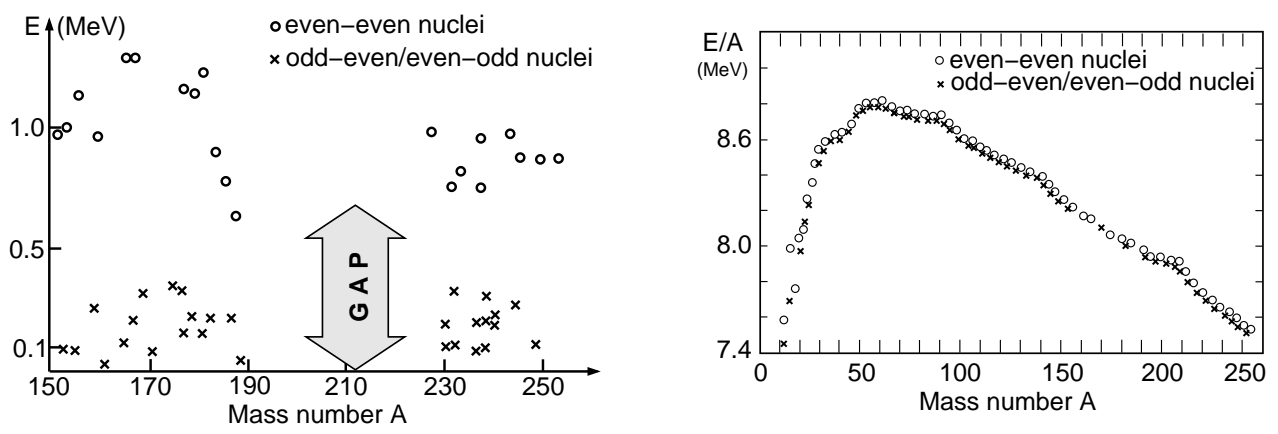

Figure 8: Left panel: lowest excitation levels of nuclei (adapted from [8]). Right panel: binding energy per nucleon for the most beta-stable isobars. 

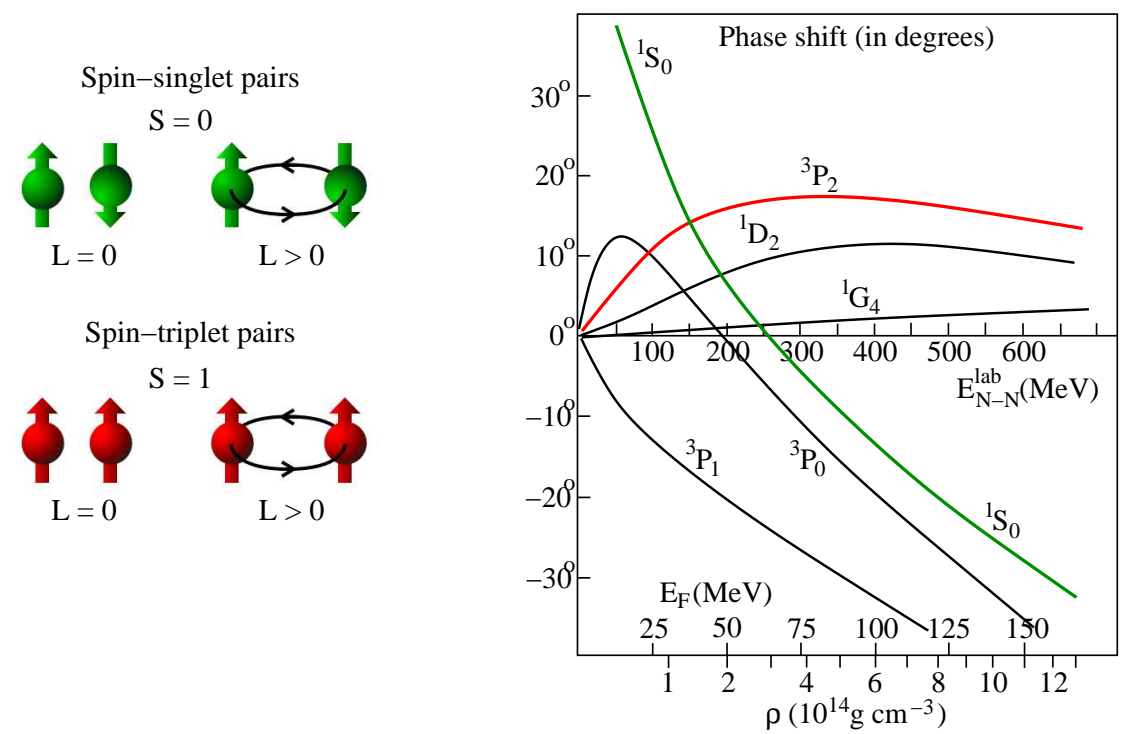

Figure 9: Left panel: possible spin-angular momentum combinations for Cooper-pairs. Right panel: phase shifts for N-N scattering as a function of the laboratory energy (middle axis) or the neutron Fermi energy and density for a neutron star interior (lower axis). Adapted from [47].

clearly require a finite minimal energy for excitation. This energy was interpreted as being the binding energy of the Cooper pair which must break to produce an excitation. In contrast, odd nuclei do not show such a gap, and this is due to the fact that they have one nucleon, neutron or proton, which is not paired and can be easily excited. The right panel of Fig. 8 shows that pairing also manifests itself in the binding energies, even-even nuclei being slightly more bound than odd nuclei ${ }^{6}$.

As a two-particle bound state, the Cooper pair can appear in many spin-orbital angular momentum states (see left panel of Fig. 9). In terrestrial superconducting metals, the Cooper pairs are generally in the ${ }^{1} \mathrm{~S}_{0}$ channel, i.e., spin-singlets with $L=0$ orbital angular momentum, whereas in liquid ${ }^{3} \mathrm{He}$ they are in spin-triplet states. What can we expect in a neutron star ? In the right panel of Fig. 9, we adapt a figure from one of the first works to study neutron pairing in the neutron star core [47] showing laboratory measured phase-shifts from N-N scattering. A positive phase-shift implies an attractive interaction. From this figure, one can expect that nucleons could pair in a spin-singlet state, ${ }^{1} \mathrm{~S}_{0}$, at low densities, whereas a spin-triplet, ${ }^{3} \mathrm{P}_{2}$, pairing should appear at higher densities. We emphasize that this is only a presumption as medium effects can strongly affect particle interactions.

A simple model can illustrate the difficulty in calculating pairing gaps. Consider a dilute Fermi gas with a weak, attractive, interaction potential $U$. The interaction is then entirely described by the corresponding scattering length, $a,{ }^{7}$ which is negative for an attractive potential. In this case,

\footnotetext{
${ }^{6}$ Notice that, as a result of pairing, the only stable odd-odd nuclei are ${ }^{2} \mathrm{H}(1,1),{ }^{6} \mathrm{Li}(3,3),{ }^{10} \mathrm{~B}(5,5)$, and ${ }^{14} \mathrm{~N}(7,7)$. All heavier odd-odd nuclei are beta-unstable and decay into an even-even nucleus.

${ }^{7}$ The scattering length $a$ is related to $U$ by $a=(m / 4 \pi \hbar) U_{0}$ with $U_{\mathbf{k}}=\int d^{3} r \exp (i \mathbf{k} \cdot \mathbf{r}) U(\mathbf{r})$.
} 

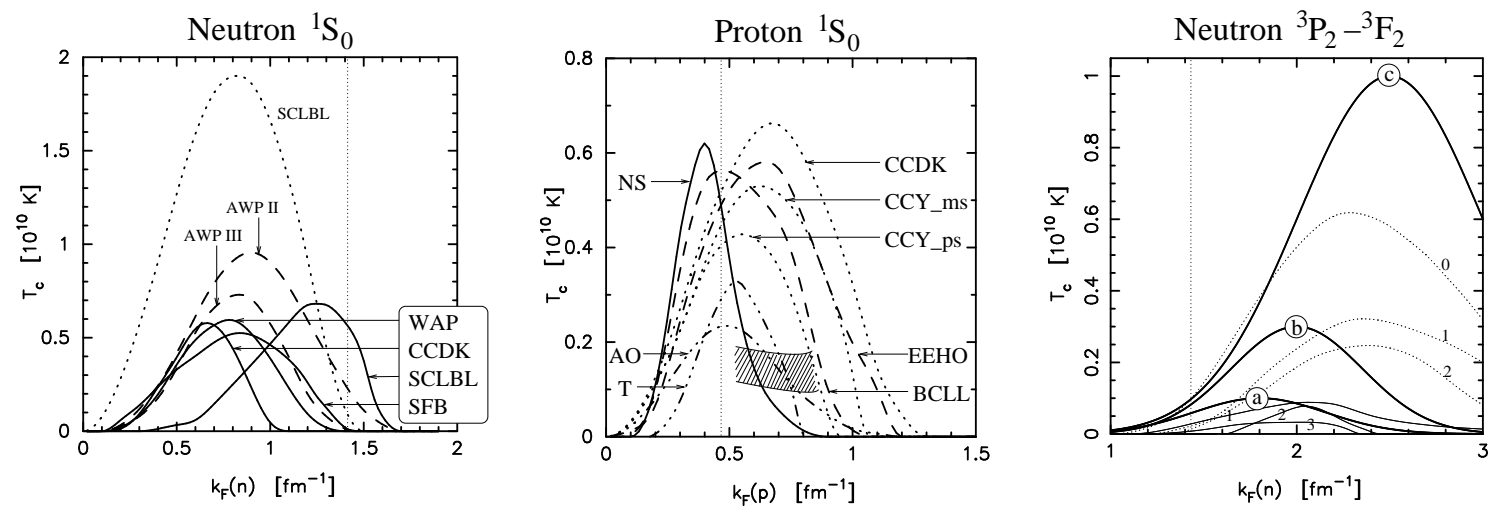

Figure 10: Three collections of predicted pairing critical temperatures for neutrons in the ${ }^{1} \mathrm{~S}_{0}$ and ${ }^{3} \mathrm{P}_{2}$ channels and protons in the ${ }^{1} \mathrm{~S}_{0}$ channel. See [35] for references to the original works.

the model has a single dimensionless parameter, $|a| k_{F}$, and the dilute gas corresponds to $|a| k_{F} \ll 1$. Assuming the pairing interaction is just the bare interaction $U$ (which is, improperly, called the $B C S$ approximation), the gap equation at $T=0$ can be solved analytically, giving the weak-coupling BCS-approximation gap:

$$
\Delta\left(k_{F}\right) \longrightarrow \Delta_{B C S}\left(k_{F}\right)=\frac{8}{e^{2}}\left(\frac{\hbar^{2} k_{F}^{2}}{2 M}\right) \exp \left[-\frac{\pi}{2|a| k_{F}}\right] \quad \text { when } \quad|a| k_{F} \rightarrow 0 .
$$

This result is bad news: the gap depends exponentially on the pairing potential $U$. The Cooper pairs have a size of the order of $\xi \sim \hbar v_{F} / \Delta$ (the coherence length) and thus $\xi k_{F} \sim \exp \left[+\pi / 2|a| k_{F}\right] \gg 1$ in the weak coupling limit. There appear to be many other particles within the pair's coherence length. These particles will react, and can screen or un-screen, the interaction. Including this medium polarization on the pairing is called beyond BCS, and in the weak coupling limit its effect has been calculated analyticaly [18], giving

$$
\Delta\left(k_{F}\right) \longrightarrow \Delta_{G M B}\left(k_{F}\right)=\frac{1}{(4 \mathrm{e})^{1 / 3}} \Delta_{B C S}\left(k_{F}\right) \simeq 0.45 \Delta_{B C S}\left(k_{F}\right) \quad \text { when } \quad|a| k_{F} \rightarrow 0 .
$$

So, screening by the medium reduces the gap by more than a factor two, even in an extremely dilute system.

Pairing correlations in nuclei are part of everyday nuclear physics, and a significant amount of work has also been devoted to the neutron star environment (see, e.g., [13] and [28] for reviews). We show in Fig. 10 three sets of predicted $T_{c}$ for the neutron star interior. At low density, corresponding to the neutron star crust in the regime of dripped neutrons, the expectation of a neutron ${ }^{1} \mathrm{~S}_{0}$ superfluid is amply confirmed by the models. This regime was also illustrated in the inset $\mathrm{A}$ of Fig. 1. At higher densities, corresponding to the neutron star core, the situation is much more ambiguous. Due to their low concentrations, protons have small Fermi momenta in the core and are expected to form a ${ }^{1} \mathrm{~S}_{0}$ superconductor. There is, however, a significant uncertainty in the size of their gap, with predicted values of $T_{c}$ ranging from $\sim 10^{9} \mathrm{~K}$ up to $6 \times 10^{9} \mathrm{~K}$, and a larger uncertainty in the range of Fermi momenta in which $\Delta\left(k_{F}\right)$ is non-zero, which translates into an uncertainty of a factor of more than 3 on the density range covered by the superconductor. In the 
"pessimistic" case protons would be superconducting only in the outer part of the core, whereas in the "optimistic" case the whole core may be superconducting.

For neutrons in the neutron star core, there is no agreement between the many published models about either the maximum value of $T_{c}$ or on the density range in which pairing is significant. Notice that, due to the tensor interaction, pairing is expected to be in the ${ }^{3} \mathrm{P}_{2}-{ }^{3} \mathrm{~F}_{2}$ channel. However, even the best models of the N-N interaction in vacuum fail to reproduce the measured phase shift in the ${ }^{3} \mathrm{P}_{2}$ channel [4]. Due to medium polarization a long-wavelength tensor force appears that is not present in the in vacuum interaction and results in a strong suppression of the gap [45]. Recently, the effect of three-body forces (TBF), absent in the laboratory $\mathrm{N}-\mathrm{N}$ scattering experiment, has been considered. TBF are necessary to reproduce the nuclear saturation density; they are, in the bulk, repulsive and their importance grows with increasing density. However, it was found in $[57,58]$ that, at the Fermi surface, they are strongly attractive in the ${ }^{3} \mathrm{P}_{2}-{ }^{3} \mathrm{~F}_{2}$ channel and result in very large neutron ${ }^{3} \mathrm{P}_{2}-{ }^{3} \mathrm{~F}_{2}$ gaps. Other delicate issues are the effect of the proton contaminant and the likely development of a $\pi^{0}$ condensate ${ }^{8}$ which also strongly affects the size of the neutron (and proton) gap(s). In short, the size and extent in density of the neutron ${ }^{3} \mathrm{P}_{2}-{ }^{3} \mathrm{~F}_{2}$ gap in the neutron star core is poorly known.

\subsection{The Cooper pair neutrino process}

The formation of the fermonic pair condensate also triggers a new neutrino emission process, which has been termed as the "pair breaking and formation", or PBF, process [44]. When an ff pair ( $\mathrm{f}=\mathrm{n}, \mathrm{p}$, or any fermion undergoing pair condensation) forms, its binding energy can be emitted as a $v-\bar{v}$ pair. Under the right conditions, this PBF process can be the dominant cooling agent in the evolution of a neutron star [32]. Such efficiency is due to the fact that the pairing phase transition is second order in nature. During the cooling of the star, the phase transition starts when the temperature $T$ reaches $T_{c}$ when pairs begin to form, but thermal agitation will constantly induce the breaking of pairs with subsequent re-formation and possible neutrino pair emission.
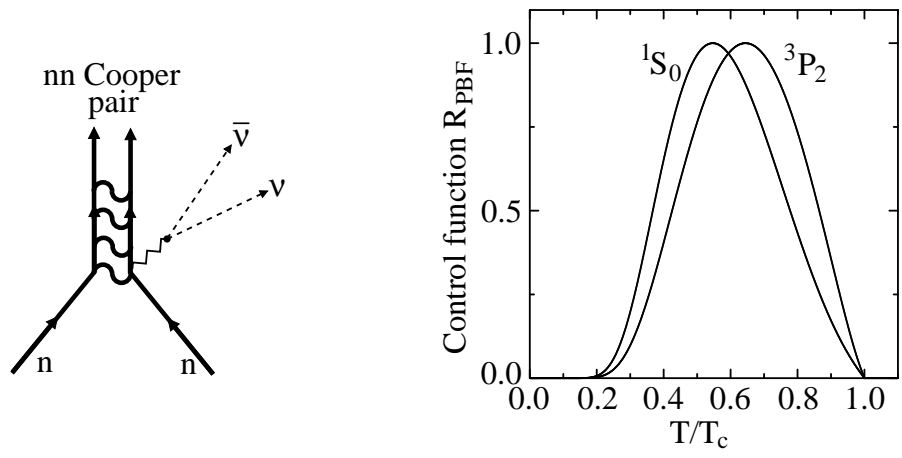

Figure 11: Left panel: Feynman diagram for $v-\bar{v}$ emission from the pair breaking and formation (PBF) process. Right panel: control functions $R_{\mathrm{PBF}}$ for the PBF process.

\footnotetext{
${ }^{8}$ In the presence of a charged $\pi^{-}$condensate a new Urca neutrino emission pathway is open, see Table 1 and Eq (3.3). The development of a neutral $\pi^{0}$ condensate has, however, little effect on neutrino emission.
} 
The emissivity of the PBF process can be written as

$$
\begin{array}{r}
\varepsilon^{\mathrm{PBF}}=\frac{12 G_{F}^{2} m_{f}^{*} p_{F, f}}{15 \pi^{5} \hbar^{10} c^{6}}\left(k_{B} T\right)^{7} a_{f, j} R_{j}\left[\Delta_{j}(T) / T\right] \\
=3.51 \times 10^{21} \frac{\mathrm{erg}}{\mathrm{cm}^{3} \mathrm{~s}} \times \tilde{m}_{f} \tilde{p}_{F, f} T_{9}^{7} a_{f, j} R_{j}\left[\Delta_{j}(T) / T\right]
\end{array}
$$

for a fermion $f$ in a pairing state $j={ }^{1} \mathrm{~S}_{0}$ or ${ }^{3} \mathrm{P}_{2}$. The coefficients $a_{f, j}$ depend on the type of fermion and on vector and axial couplings $C_{V}$ and $C_{A}$ (see, e.g., [36]). The control functions $R_{j}$ are plotted in the right panel of Fig. 11. These functions encapsulate the effect that the PBF process turns on when $T$ reaches $T_{c}$ and practically turns off at $T \lesssim 0.2 T_{c}$ when there is not enough thermal energy to break pairs. The PBF process was first discovered by Flowers, Ruderman, and Sutherland [15] and, independently, by Voskresensky and Senatorov [51]. It was, however, completely overlooked for 20 years (?) until finally implemented in a cooling calculation in [44] and its importance emphasized in [32].

An alternative way of looking at the PBF process is simply as an inter band transition of a nucleon [55]. Considering the particle spectrum in a paired state, right panel of Fig. 5, the lower branch (with $\varepsilon<\varepsilon_{F}-\Delta$ ) corresponds to paired particles whereas the upper branch to excited ones, i.e., coming from a "broken pair" which hence left a hole in the lower branch. A transition of a particle from the upper branch to a hole in the lower branch corresponds to the formation of a Cooper pair.

\section{Cooling of Superfluid Neutron Stars}

In order to complement the brief description of cooling presented in $\S 4.4$, we now include the effects of pairing, but restrict ourself to cases in which no fast neutrino emission process is allowed (the minimal cooling scenario [35, 36]). In the left panel of Fig. 12, three examples of the thermal evolution of a $1.4 M_{\odot}$ star are shown. The 4 cooling stages, introduced in Fig. 4 , are similarly marked in this figure. The first model does not include any effect from pairing, and is very similar to the $1.4 M_{\odot}$ model of Fig. 4 (but not completely identical because of several improvements in the microphysics with respect to the older calculations of [33]). The other two models include nucleon pairing and in both cases the suppressing effects on the neutrino emission as well as the modification of the specific heat are taken into account (see Eqs. (5.3) and (5.2)), but the PBF process is artificially turned off in one case. When Cooper pair neutrino emission from the PBF process is turned off, one can clearly see the effect of the suppression of the modified Urca and bremsstrahlung processes during stage 3 which results in a much warmer star. One can also see the effect of the suppression of the specific heat which results in a faster cooling during the photon cooling era (stage 4). During stage 3, the specific heat is also suppressed, but its effect is not as pronounced as in stage 4. In the presence of the PBF process, however, the star is significantly colder during stage 3 , the PBF process being more efficient than the MU process in the unsuppressed case (i.e., no pairing). In stage 4, the two trajectories with pairing, but with and without the PBF process, converge as cooling is now driven by the photons and only the specific heat suppression by pairing is relevant. Notice that during stage 2, the crust relaxation period, the model with pairing (and PBF) has a shorter relaxation time because of the suppression of the 

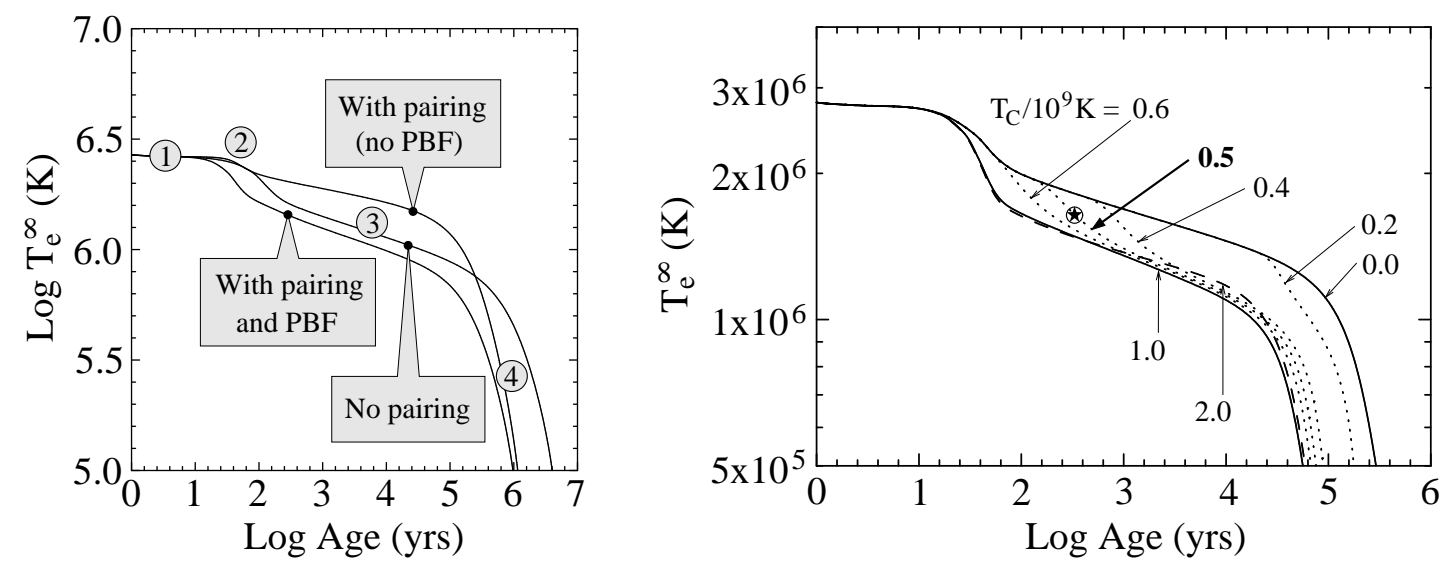

Figure 12: Left panel: three models of cooling neutron stars illustrating the impact of pairing. Right panel: exploration of the effect of a late onset of neutron superfluidity [36, 37]. The curves are labeled according to the maximum value of the neutron ${ }^{3} \mathrm{P}_{2} T_{c}$ : $T_{C}=\max T_{c}\left(k_{F}\right)$. No proton superconductivity is taken into account in these models. The star marks the observed value of $T_{e}^{\infty}$ for the Cas A neutron star at an age of 330 yrs. See text for description.

neutron specific heat from the ${ }^{1} \mathrm{~S}_{0}$ gap in the inner crust (the same effect is present in the model with pairing and no PBF, but is barely visible because the core is too hot). In stage 1, the three models give identical $T_{e}$ 's as at that age $T_{e}$ is controlled by the evolution of the upper layers where no superfluid is present.

The constituent whose pairing has the major effect is the neutron fluid in the core. For illustrative purposes, the left panel of Fig. 12 employed a neutron ${ }^{3} \mathrm{P}_{2}$ gap chosen to maximize the differences between the three models: the bell shaped $T_{c}\left(k_{F}\right)$ curve (see the examples in Fig. 10) reaches a maximum of $T_{C} \equiv T_{c}^{\max } \simeq 5 \times 10^{9} \mathrm{~K}$. The right panel of Fig. 12 shows a series of models in which the neutron ${ }^{3} \mathrm{P}_{2}$ gap $T_{c}\left(k_{F}\right)$ is rescaled by a constant factor, keeping the same shape. The dotted curves, with low values of $T_{C}$, exhibit a transit of the neutron star from the warm trajectory with $T_{C}=0$ toward the cold trajectories, with $T_{C}=1$ or $2 \times 10^{9} \mathrm{~K}$. At early times when the star's core temperature $T$ is above $T_{C}$, neutrons are normal but when $T$ reaches $T_{C}$ the phase transition starts at some location in the star (see the left panel of Fig. 13 for a schematic of the evolution of $L_{v}$ ). At that stage, neutrons in a thick shell go through the phase transition and the neutrino luminosity suddenly increases due to the triggering of the PBF process. The star then begins its transit toward a colder trajectory. As $T$ decreases, this shell splits into two shells which slowly drift toward the lower and higher density regions away from the maximum of the bell-shaped $T_{c}\left(k_{F}\right)$ profile. When $T$ is much below $T_{c}\left(k_{F}\right)$ everywhere in the core, $L_{v}$ decreases rapidly. In the cases for which $T_{C} \lesssim 8 \times 10^{8} \mathrm{~K}$ the onset of the phase transition occurs after the period of thermal relaxation of the crust and is seen as a second phase of rapid decrease of $T_{e}$. For larger values of $T_{C}$, this transitory rapid cooling of the core is hidden by the crust which is not yet thermally relaxed with the core.

The simple analytical solution of Eq. (4.9) gives some insight into this transitory behavior. When $T>T_{C}$, but $\ll T_{0}$, the star follows the asymptotic "MU trajectory", $T \sim t^{-1 / 6}$, and when $T$ reaches $T_{C}$, at time $t=t_{C}$, the neutrino luminosity suddenly increases. Despite the complicated $T$ dependence of $\varepsilon^{\mathrm{PBF}}$, Eq. (5.6), the resulting luminosity, once integrated over the entire core 

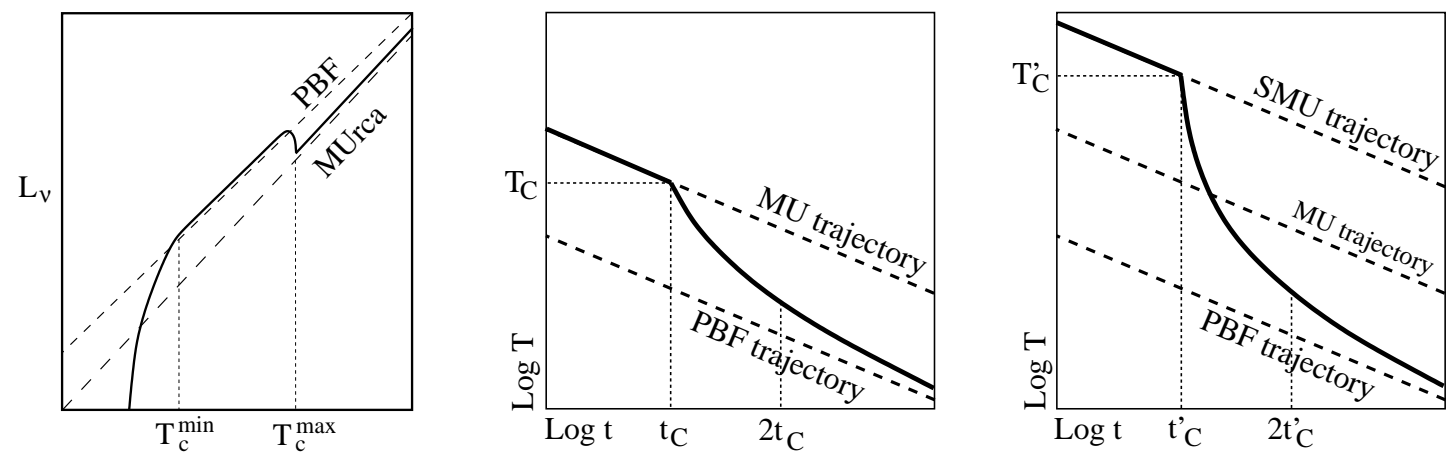

Figure 13: See text for description. Figure from [36] and [37].

(also, aided the bell shape of the $T_{c}\left(k_{F}\right)$ curve), is well approximated by a $T^{8}$ power law in the $T$ regime in which some thick shell of neutrons is going through the phase transition. If we write $L_{v}^{\mathrm{PBF}}=f \cdot L_{v}^{\mathrm{MU}}=f N_{9} T_{9}^{8}$, with $f \sim 10$, the solution of Eq. (4.9), replacing $t_{0}$ by $t_{C}$ and $T_{0}$ by $T_{C}$, gives

$$
T=\frac{T_{C}}{\left[1+f\left(t-t_{C}\right) / t_{C}\right]^{1 / 6}} \quad \text { and } \quad T=10^{9} \mathrm{~K}\left(\tau_{\mathrm{MU}} / f t\right)^{1 / 6}\left(\text { when } T \ll T_{C}\right) .
$$

The central panel of Fig. 12 shows the two asymptotic MU and PBF trajectories and the transit trajectory. Notice that in case the neutrino emission was already suppressed previously to the onset of neutron superfluidity, e.g., by proton superconductivity with a larger critical temperature, the MU trajectory is replaced by a warmer suppressed MU (SMU) trajectory and the initial part of the transit is much faster, as illustrated in the right panel of Fig. 12.

\section{Cassiopeia A and its Cooling Neutron Star}

The Cassiopeia A supernova remnant (SNR) was discovered in radio in 1947 and is the second brightest radio source in the sky (after the Sun). It has since then been observed at almost all wavelengths. Very likely, this supernova was observed by the first Astronomer Royal, John Flamsteed [2] who, on August 16, 1680, when describing the stars in Cassiopeia constellation listed the star "3 Cassiopeia" at a position almost coincident with the supernova remnant. This star had never been reported previously, and was never seen again until August 1999 when the first light observation of Chandra found a point source in the very center of the remnant (see Fig. 14).

The distance to the SNR is $3.4_{-0.1}^{+0.4} \mathrm{kpc}$ [43], and the direct observation, by the Hubble Space Telescope, of the remnant expansion implies a birth in the second half of the $17^{\text {th }}$ century [14] and supports Flamsteed's observation. These observations give a present age of 331 yrs for the neutron star in Cassiopeia A. The optical spectrum of the supernova has been observed through its light echo from scattering of the original light by a cloud of interstellar dust and shows the supernova was of type IIb [24]. The progenitor was thus a red supergiant that had lost most of its hydrogen envelope, with an estimated zero age main sequence (ZAMS) mass of 16 to $20 M_{\odot}$ [52, 11, 49] or even up to $25 M_{\odot}$ in the case of a binary system [56]. This implies a relatively massive neutron star, i.e. likely $\gtrsim 1.4 M_{\odot}[56]$. 


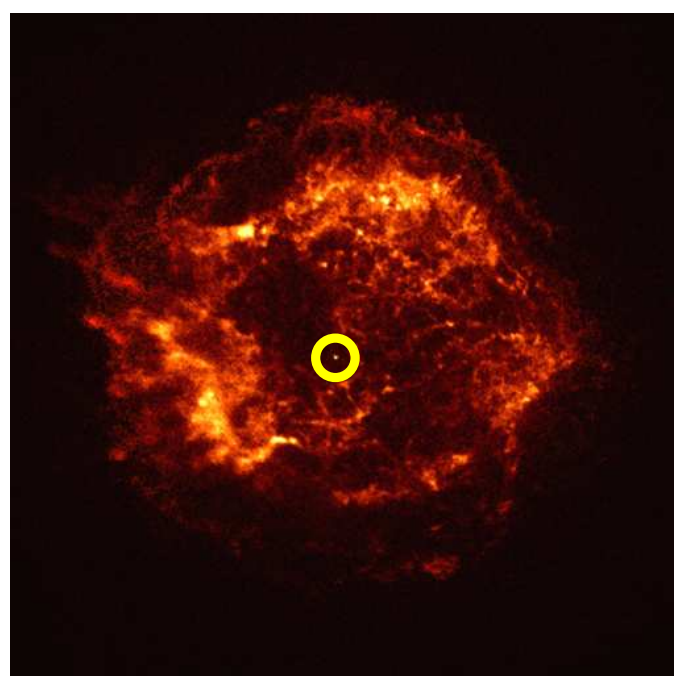

Figure 14: The Cassiopeia supernova remnant in X-rays: first light of Chandra, August 1999. (The neutron star is highlighted by the authors.) Image from (C) NASA/CXC/SAO.

The soft X-ray spectrum of the point source in the center of the SNR in Cassiopeia A is thermal, but its interpretation has been challenging [39]. With a known distance, a measurement of the temperature implies a measurement of the star's radius, but spectral fits with a blackbody or a $\mathrm{H}$ atmosphere model resulted in an estimated radius of 0.5 and $2 \mathrm{~km}$, respectively. It was only in 2009 that a successful model was found: a non-magnetized ${ }^{9} \mathrm{C}$ atmosphere, which implies a stellar radius between 8 to $18 \mathrm{~km}$ [22]. With this model, and analyzing 5 observations of the SNR, Heinke $\&$ Ho [20] found that the neutron star temperature had dropped by 4\% from 2000 to 2009, from 2.12 to $2.04 \times 10^{6} \mathrm{~K}$, and the observed flux had decreased by $21 \%$. The neutron star in Cassiopeia $\mathrm{A}$ is thus the youngest known neutron star and the first one whose cooling has been observed in real time!

\section{Superfluid Neutrons in the Core of the Cassiopeia A Neutron Star}

The Chandra observations of Cassiopeia A presented in the previous section, together with its known distance, imply that the photon luminosity of the neutron star is

$$
L_{\gamma} \simeq 10^{34} \mathrm{erg} \mathrm{s}^{-1}
$$

With a measured $T_{e} \simeq 2 \times 10^{6} \mathrm{~K}$ [22], we deduce an internal $T \simeq 4 \times 10^{8} \mathrm{~K}$ from Eq. (4.7). The star's total specific heat is thus $C_{v} \simeq 4 \times 10^{38} \mathrm{erg} \mathrm{K}{ }^{-1}$ (from Fig. 3 or Eq. (4.8)). The observed $\Delta T_{e} / T_{e} \simeq 4 \%$ [20] gives for the internal temperature $\Delta T / T \simeq 8 \%$ over a ten years period since $T \sim T_{e}^{2}$. Assuming the observed cooling corresponds to a global cooling of the whole neutron star, its thermal energy loss is

$$
\dot{E}_{\mathrm{th}}=C_{V} \dot{T} \simeq\left(4 \times 10^{38} \mathrm{erg} \mathrm{K}^{-1}\right) \times\left(0.1 \mathrm{~K} \mathrm{~s}^{-1}\right) \simeq 4 \times 10^{37} \mathrm{erg} \mathrm{s}^{-1}
$$

\footnotetext{
${ }^{9}$ There is, to date, no evidence for the presence of a magnetic field in the Cassiopeia A neutron star.
} 


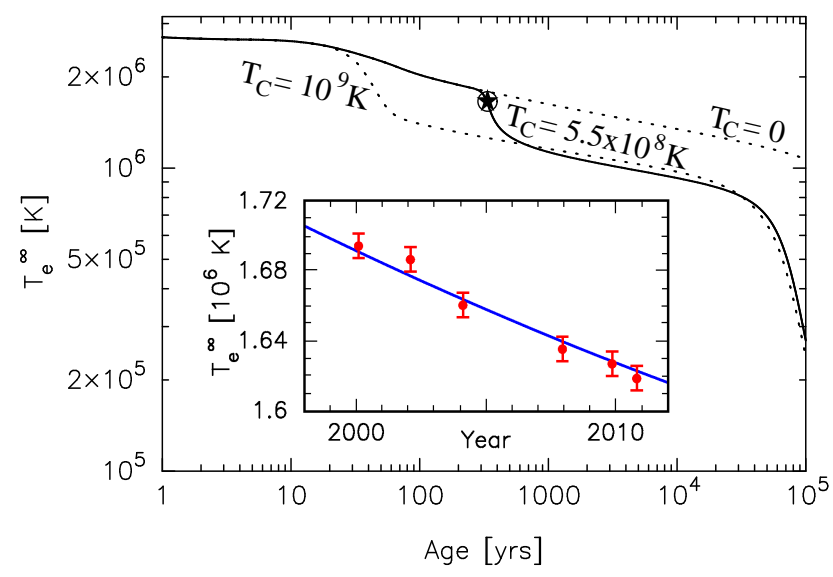

Figure 15: A very good fit of the rapid cooling of the neutron star in Cassiopeia A obtained assuming a recent onset of neutron ${ }^{3} \mathrm{P}_{2}$ superfluidity and the resulting increase in neutrino emission from the formation and breaking of Cooper pairs. The successful model assumes a maximum critical temperature $T_{C}=5.5 \times 10^{8}$ $\mathrm{K}$ and the inset compares it with the six observational points, with their $1 \sigma$ error bars, from [20] and [46]. The two dotted curves with no neutron superfluidity, $T_{C}=0$, and superfluidity with a higher $T_{C}=1 \times 10^{9} \mathrm{~K}$ illustrate the sensitivity to $T_{C}$. Figure adapted from [37].

which is 3-4 orders of magnitude larger than what it seen in $L_{\gamma}$ ! For a young neutron star, neutrinos are the prime candidates to induce such a large energy loss.

The cooling rate of this neutron star is so large that it must be a transitory event, which was initiated only recently. Something critical occurred recently within this star! "Something critical" for a cooling neutron star points toward a critical temperature, and a phase transition is a good candidate. The results of the previous section exhibited a phase of accelerated cooling when the neutron ${ }^{3} \mathrm{P}_{2}$ pairing phase transition is triggered. With a $T_{C} \simeq 5 \times 10^{8} \mathrm{~K}$, a transitory cooling can occur at an age $\simeq 300$ yrs as shown in the right panel of Fig. 12 .

This interpretation of the observed rapid cooling of the neutron star in Cassiopeia A as triggered by the recent onset of the neutron ${ }^{3} \mathrm{P}_{2}$ superfluid phase transition and the resulting increase in neutrino emission from the formation and breaking of pairs in the neutron superfluid was recently proposed in [37] and, independently, in [46]. Models such as the ones in the right panel of Fig. 12 do not, however, exhibit a cooling rate as large as the observed one.

A second key ingredient for reproducing the observed cooling rate is illustrated in the right panel of Fig. 13. The neutron star was very hot before the onset of neutron superfluidity. This is possible in the case the protons were already in a superconducting state, which implies that the corresponding critical temperature is significantly larger than the $T_{c}$ for neutrons. That the $T_{c}$ for ${ }^{1} \mathrm{~S}_{0}$ proton pairing is larger than the $T_{c}$ for neutron ${ }^{3} \mathrm{P}_{2}$ pairing is expected from the theoretical results presented in $\$ 5.1$. However, a hot young neutron star can only be achieved in the case that the protons are superconducting in the entire core, so that neutrino emission form the modified Urca processes is strongly suppressed [46, 37]. This requirement places strong constraints on the proton ${ }^{1} \mathrm{~S}_{0}$ pairing and is easier to fulfill if the neutron star mass is not too large as theoretical models show that proton superconductivity does not extend to very high densities. A better understanding of the progenitor of Cassiopeia A, and constraining the expected neutron star mass, is essential for 
this scenario to work.

A very good fit to the observations is shown in Fig. 15, in which a maximum neutron ${ }^{3} \mathrm{P}_{2}$ pairing $T_{C}$ of $5.5 \times 10^{8} \mathrm{~K}$ is employed and protons are assumed to be superconducting in the entire core with $T_{c}\left(k_{F}\right)>10^{9} \mathrm{~K}$ everywhere. Very similar results were obtained independently in [46].

\section{Discussion and Conclusions}

The observation, in real time, of the cooling of a neutron star is unique and its interpretation potentially imposes very strong constraints on the physics of ultra-dense matter. We have here given a basic presentation of the physical principles that are involved in trying to understand the interior of a neutron star. Neutrino emission processes and the likely occurrence of pairing are the two most important ingredients, and in both cases many unsettled issues still remain.

We have presented arguments which, we hope, make it plausible that the observed $4 \%$ temperature drop, in a period of 10 years, of the young neutron star in the Cassiopeia A supernova remnant may signal the recent triggering of the phase transition of the core neutron fluid to a superfluid state. The resulting on-going formation of ${ }^{3} \mathrm{P}_{2}$ Cooper pairs results in a strong neutrino emission which can explain the observed rapid temperature drop of this neutron star. An essential requirement for the cooling to be as rapid as observed is that the neutron star was relatively hot at earlier times, and we described how this may be due to the suppression of the early neutrino emission by proton superconductivity. This would imply that the critical temperature, $T_{c}^{\mathrm{p}}$, for proton superconductivity is, everywhere in the star's core, larger than $10^{9} \mathrm{~K}$. Under this condition, we found that the critical temperature for neutron superfluidity, which is density dependent, must have a maximum value of $T_{C}^{\mathrm{n}} \simeq 5 \times 10^{8} \mathrm{~K}$. This would be the first direct evidence that neutron and proton superfluidity/superconductivity occur at supra-nuclear densities in the core of a neutron star $[37,46]$ and, further, these results would highly constrain their respective critical temperatures.

Alternatively, one could consider the observed cooling of the star in Cassiopeia A to be instead due to a longer thermal relaxation timescale in some layer of the star than we have assumed. In such a case the estimate of Eq. (8.2), which assumes an isothermal star, does not apply. As seen, e.g., in Fig. 12, the crust thermal relaxation occurs on a time-scale of a few decades. However, in case the crust thermal conductivity is smaller that the one employed here it may possible that this early temperature drop be delayed till the star is 300-400 years old (see, e.g., [53]). Similarly, the core thermal relaxation time may be much larger than usually considered: in case the inner core of the star cools rapidly it may take a few hundreds years for the star to become isothermal and this can induce a rapid decrease of $T_{e}$, as proposed in [7] and presented in these proceedings by $\mathrm{D}$. Blaschke. This scenario requires that the core thermal conductivity be lower than usually considered, by a factor of a few, and also implies that neutrons do not form a superfluid until the star is much colder.

The neutron superfluid scenario we have presented fits within the minimal cooling scenario [35] and is compatible with observations of other cooling neutron stars [36]. The very similar model of [46] also successfully passes the same test as well as the opposite core relaxation scenario of [7]. More work is required to confront these possibilities with other facets of the neutron star phenomenology. 


\section{Acknowledgments}

DP's acknowledges support by grants from UNAM-DGAPA, \# PAPIIT-113211, and Conacyt, CB-2009-01, \#132400, and thanks the organizers of the XXXIV Brazilian Workshop on Nuclear Physics for the generous invitation. MP and JML acknowledge research support from the U.S. DOE grants DE-FG02-93ER-40756 and DE-AC02-87ER40317, respectively. AWS is supported by Chandra grant TM1-12003X, by NSF PHY grant 08-22648, by NASA ATFP grant NNX08AG76G, and by DOE grant DE-FG02-00ER41132.

\section{References}

[1] A. Akmal, V. R. Pandharipande, and D. G. Ravenhall, Equation of state of nucleon matter and neutron star structure, Phys. Rev. C58 (1998), 1804-1828.

[2] W. B. Ashworth, Jr., A Probable Flamsteed Observations of the Cassiopeia-A Supernova, Journal for the History of Astronomy 11 (1980), 1-+.

[3] Baade, W. and Zwicky, F., Cosmic rays from super-novae, Proceedings of the National Academy of Science 20 (1934), 259-263.

[4] M. Baldo, Ø. Elgarøy, L. Engvik, M. Hjorth-Jensen, and H.-J. Schulze, ${ }^{3} P_{2}-{ }^{3} F_{2}$ pairing in neutron matter with modern nucleon-nucleon potentials, Phys. Rev. C58 (1998), 1921-1928.

[5] J. Bardeen, L. N. Cooper, and J. R. Schrieffer, Theory of Superconductivity, Physical Review 108 (1957), 1175-1204.

[6] G. Baym and C. Pethick, Landau fermi-liquid theory, Wiley-VCH, 2004.

[7] D. Blaschke, H. Grigorian, D. N. Voskresensky, and F. Weber, On the Cooling of the Neutron Star in Cassiopeia A, ArXiv e-prints (2011).

[8] A. Bohr, B. R. Mottelson, and D. Pines, Possible Analogy between the Excitation Spectra of Nuclei and Those of the Superconducting Metallic State, Physical Review 110 (1958), 936-938.

[9] G. E. Brown, K. Kubodera, D. Page, and P. Pizzochero, Strangeness condensation and cooling of neutron stars, Phys. Rev. D37 (1988), 2042-2046.

[10] A. Burrows and J. M. Lattimer, The birth of neutron stars, ApJ307 (1986), 178-196.

[11] R. A. Chevalier and J. Oishi, Cassiopeia A and Its Clumpy Presupernova Wind, ApJ593 (2003), L23-L26.

[12] L. N. Cooper, Bound Electron Pairs in a Degenerate Fermi Gas, Physical Review 104 (1956), 1189-1190.

[13] D. J. Dean and M. Hjorth-Jensen, Pairing in nuclear systems: from neutron stars to finite nuclei, Reviews of Modern Physics 75 (2003), 607-656.

[14] R. A. Fesen, M. C. Hammell, J. Morse, R. A. Chevalier, K. J. Borkowski, M. A. Dopita, C. L. Gerardy, S. S. Lawrence, J. C. Raymond, and S. van den Bergh, The Expansion Asymmetry and Age of the Cassiopeia A Supernova Remnant, ApJ645 (2006), 283-292.

[15] E. Flowers, M. Ruderman, and P. Sutherland, Neutrino pair emission from finite-temperature neutron superfluid and the cooling of young neutron stars, ApJ205 (1976), 541-544. 
[16] E. G. Flowers, P. G. Sutherland, and J. R. Bond, Neutrino pair bremsstrahlung by nucleons in neutron-star matter, Phys. Rev. D12 (1975), 315-318.

[17] B. L. Friman and O. V. Maxwell, Neutrino emissivities of neutron stars, ApJ232 (1979), 541-557.

[18] L. P. Gorkov and T. K. Melik-Barkhudarov, Contribution to the Theory of Superfluidity in an Imperfect Fermi Gas, Sov. Phys. JETP 13 (1961), 1018.

[19] E. H. Gudmundsson, C. J. Pethick, and R. I. Epstein, Structure of neutron star envelopes, ApJ272 (1983), 286-300.

[20] C. O. Heinke and W. C. G. Ho, Direct Observation of the Cooling of the Cassiopeia A Neutron Star, ApJ719 (2010), L167-L171.

[21] J. W. T. Hessels, S. M. Ransom, I. H. Stairs, P. C. C. Freire, V. M. Kaspi, and F. Camilo, A Radio Pulsar Spinning at 716 Hz, Science 311 (2006), 1901-1904.

[22] W. C. G. Ho and C. O. Heinke, A neutron star with a carbon atmosphere in the Cassiopeia A supernova remnant, Nature462 (2009), 71-73.

[23] N. Iwamoto, Neutrino emissivities and mean free paths of degenerate quark matter., Annals of Physics 141 (1982), 1-49.

[24] O. Krause, S. M. Birkmann, T. Usuda, T. Hattori, M. Goto, G. H. Rieke, and K. A. Misselt, The Cassiopeia A Supernova Was of Type IIb, Science 320 (2008), 1195-.

[25] D. Lai, Matter in strong magnetic fields, Reviews of Modern Physics 73 (2001), 629-+.

[26] J. M. Lattimer and M. Prakash, Ultimate Energy Density of Observable Cold Baryonic Matter, Physical Review Letters 94 (2005), no. 11, 111101-+.

[27] J. M. Lattimer, M. Prakash, C. J. Pethick, and P. Haensel, Direct URCA process in neutron stars, Physical Review Letters 66 (1991), 2701-2704.

[28] U. Lombardo and H.-J. Schulze, Superfluidity in Neutron Star Matter, Physics of Neutron Star Interiors (D. Blaschke, N. K. Glendenning, \& A. Sedrakian, ed.), Lecture Notes in Physics, Berlin Springer Verlag, vol. 578, 2001, pp. 30-+.

[29] O. Maxwell, G. E. Brown, D. K. Campbell, R. F. Dashen, and J. T. Manassah, Beta decay of pion condensates as a cooling mechanism for neutron stars, ApJ216 (1977), 77-85.

[30] A. Migdal, Superfluidity and the moments of inertia of nuclei, Nucl. Phys. A13 (1959), 655-674.

[31] J. R. Oppenheimer and G. M. Volkoff, On Massive Neutron Cores, Physical Review 55 (1939), 374-381.

[32] D. Page, Thermal Evolution of Isolated Neutron Stars, NATO ASIC Proc. 515: The Many Faces of Neutron Stars. (R. Buccheri, J. van Paradijs, \& A. Alpar, ed.), 1998, pp. 539-+.

[33] D. Page and J. H. Applegate, The cooling of neutron stars by the direct URCA process, ApJ394 (1992), L17-L20.

[34] D. Page, U. Geppert, and F. Weber, The cooling of compact stars, Nuclear Physics A 777 (2006), 497-530.

[35] D. Page, J. M. Lattimer, M. Prakash, and A. W. Steiner, Minimal Cooling of Neutron Stars: A New Paradigm, ApJS155 (2004), 623-650.

[36] Neutrino Emission from Cooper Pairs and Minimal Cooling of Neutron Stars, ApJ707 (2009), 1131-1140. 
[37] D. Page, M. Prakash, J. M. Lattimer, and A. W. Steiner, Rapid Cooling of the Neutron Star in Cassiopeia A Triggered by Neutron Superfluidity in Dense Matter, Physical Review Letters 106 (2011), no. 8, 081101-+.

[38] D. Page and S. Reddy, Dense Matter in Compact Stars: Theoretical Developments and Observational Constraints, Annual Review of Nuclear and Particle Science 56 (2006), 327-374.

[39] G. G. Pavlov, D. Sanwal, and M. A. Teter, Central Compact Objects in Supernova Remnants, Young Neutron Stars and Their Environments (F. Camilo \& B. M. Gaensler, ed.), IAU Symposium, vol. 218, 2004, pp. 239-+.

[40] C. J. Pethick and A. Y. Potekhin, Liquid crystals in the mantles of neutron stars, Physics Letters B 427 (1998), 7-12.

[41] M. Prakash, J. M. Lattimer, and T. L. Ainsworth, Equation of state and the maximum mass of neutron stars, Physical Review Letters 61 (1988), 2518-2521.

[42] M. Prakash, M. Prakash, J. M. Lattimer, and C. J. Pethick, Rapid cooling of neutron stars by hyperons and Delta isobars, ApJ390 (1992), L77-L80.

[43] J. E. Reed, J. J. Hester, A. C. Fabian, and P. F. Winkler, The Three-dimensional Structure of the Cassiopeia A Supernova Remnant. I. The Spherical Shell, ApJ440 (1995), 706-+.

[44] C. Schaab, D. Voskresensky, A. D. Sedrakian, F. Weber, and M. K. Weigel, Impact of medium effects on the cooling of non-superfluid and superfluid neutron stars., A\&A321 (1997), 591-604.

[45] A. Schwenk and B. Friman, Polarization Contributions to the Spin Dependence of the Effective Interaction in Neutron Matter, Physical Review Letters 92 (2004), no. 8, 082501-+.

[46] P. S. Shternin, D. G. Yakovlev, C. O. Heinke, W. C. G. Ho, and D. J. Patnaude, Cooling neutron star in the Cassiopeia A supernova remnant: evidence for superfluidity in the core, MNRAS412 (2011), L108-L112.

[47] R. Tamagaki, Superfluid state in neutron star matter. I. Generalized Bogoliubov transformation and existence of ${ }^{3} P_{2}$ gap at high density., Progress of Theoretical Physics 44 (1970), 905-928.

[48] D. R. Tilley and J. Tilley, Superfluidity \& superconductivity, Graduate Student Series in Physics, Institute of Physics Publishing, 1990.

[49] B. van Veelen, N. Langer, J. Vink, G. García-Segura, and A. J. van Marle, The hydrodynamics of the supernova remnant Cassiopeia A. The influence of the progenitor evolution on the velocity structure and clumping, A\&A503 (2009), 495-503.

[50] D. N. Voskresensky, Neutrino Cooling of Neutron Stars: Medium Effects, Physics of Neutron Star Interiors (D. Blaschke, N. K. Glendenning, \& A. Sedrakian, ed.), Lecture Notes in Physics, Berlin Springer Verlag, vol. 578, 2001, pp. 467-+.

[51] D. N. Voskresensky and A. V. Senatorov, Description of nuclear interaction in the keldysh diagram technique and neutrino luminosity of neutron stars, Sov. J. Nucl. Phys. 45 (1987), 411.

[52] R. Willingale, J. A. M. Bleeker, K. J. van der Heyden, and J. S. Kaastra, The mass and energy budget of Cassiopeia A, A\&A398 (2003), 1021-1028.

[53] D. G. Yakovlev, W. C. G. Ho, P. S. Shternin, C. O. Heinke, and A. Y. Potekhin, Cooling rates of neutron stars and the young neutron star in the Cassiopeia A supernova remnant, MNRAS411 (2011), 1977-1988. 
[54] D. G. Yakovlev, A. D. Kaminker, O. Y. Gnedin, and P. Haensel, Neutrino emission from neutron stars, Phys. Rep.354 (2001), 1-155.

[55] D. G. Yakovlev, A. D. Kaminker, and K. P. Levenfish, Neutrino emission due to Cooper pairing of nucleons in cooling neutron stars, A\&A343 (1999), 650-660.

[56] P. A. Young, C. L. Fryer, A. Hungerford, D. Arnett, G. Rockefeller, F. X. Timmes, B. Voit, C. Meakin, and K. A. Eriksen, Constraints on the Progenitor of Cassiopeia A, ApJ640 (2006), 891-900.

[57] X.-R. Zhou, H.-J. Schulze, E.-G. Zhao, F. Pan, and J. P. Draayer, Pairing gaps in neutron stars, Phys. Rev. C70 (2004), no. 4, 048802-+.

[58] W. Zuo, C. X. Cui, U. Lombardo, and H.-J. Schulze, Three-body force effect on ${ }^{3} P F_{2}$ neutron superfluidity in neutron matter, neutron star matter, and neutron stars, Phys. Rev. C78 (2008), no. 1, 015805-+. 